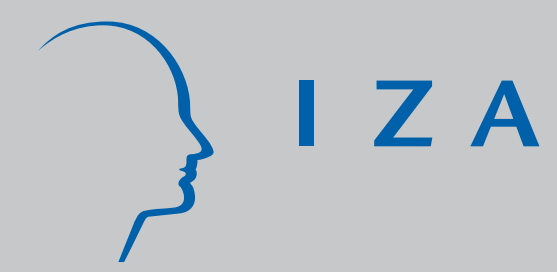

IZADP No. 1538

Limited Attention as the Scarce Resource in an Information-rich Economy

J osef Falkinger

March 2005 


\title{
Limited Attention as the Scarce Resource in an Information-Rich Economy
}

\author{
Josef Falkinger \\ University of Zurich \\ and IZA Bonn
}

\section{Discussion Paper No. 1538 \\ March 2005}

\author{
IZA \\ P.O. Box 7240 \\ 53072 Bonn \\ Germany \\ Phone: +49-228-3894-0 \\ Fax: +49-228-3894-180 \\ Email: iza@iza.org
}

\begin{abstract}
Any opinions expressed here are those of the author(s) and not those of the institute. Research disseminated by IZA may include views on policy, but the institute itself takes no institutional policy positions.

The Institute for the Study of Labor (IZA) in Bonn is a local and virtual international research center and a place of communication between science, politics and business. IZA is an independent nonprofit company supported by Deutsche Post World Net. The center is associated with the University of Bonn and offers a stimulating research environment through its research networks, research support, and visitors and doctoral programs. IZA engages in (i) original and internationally competitive research in all fields of labor economics, (ii) development of policy concepts, and (iii) dissemination of research results and concepts to the interested public.
\end{abstract}

IZA Discussion Papers often represent preliminary work and are circulated to encourage discussion. Citation of such a paper should account for its provisional character. A revised version may be available directly from the author. 
IZA Discussion Paper No. 1538

March 2005

\section{ABSTRACT \\ Limited Attention as the Scarce Resource in an Information-rich Economy*}

This paper uses basic empirical facts from attention and perception psychology for a behavioral approach to equilibrium analysis at the industry and the macroeconomic level. The paper endogenously determines whether an economy is information-rich and whether scarcity of attention complements economic scarcity. A conventional economic equilibrium results if subjects have free attention capacity. At the positive level, the impacts of ITprogress, international integration and media on equilibrium diversity and level of attentionseeking activities are shown. At the normative level, welfare, efficiency and optimal policy interventions are characterized. Finally, behavioral effects of intensified attention-seeking on market power, sectoral economic structure and work-leisure choice are considered.

JEL Classification: D50, D80, L10

Keywords: limited attention, information-rich economies, media, diversity, behavioral equilibrium analysis

Corresponding author:

Josef Falkinger

University of Zurich

Socioeconomic Institute

Zürichbergstrasse 14

$\mathrm{CH}-8032$ Zürich

Switzerland

E-mail: josef.falkinger@wwi.unizh.ch

\footnotetext{
*I wish to thank Hartmut Egger, Volker Grossmann, Michel Habib and the participants of the economic seminar at Bonn, in particular Armin Falk, for valuable hints and comments. An earlier version of the paper was presented as NÖG lecture at the Austrian Economic Society Meeting in Vienna, May 2004. I thank the participants for their discussion.
} 


\section{Introduction}

This paper presents an equilibrium model in which firms have to compete for the limited attention of consumers before they can compete for their budgets. Psychological parameters together with economic and information-technological characteristics determine whether an economy is information-poor or information-rich. In an information-poor economy, any item brought to the attention of buyers is perceived and only economic properties are decisive for its profitability. In contrast, if an economy is information-rich, the diversity of perceived items is limited and only sellers who expose consumers to sufficiently strong signals are viable. The paper shows that in an information-rich economy IT-progress, globalization or distribution of information through media channels increase the level of attention seeking activities and raise the minimal signal strength required to be attended by consumers. This can have further psychological impacts on buyer behavior which, among other things, lead in the macroeconomic equilibrium to rising price-cost margins or to a bias towards industries with strong competition for attention. Welfare is definitely higher in an information-rich than in an information-poor economy. However, whereas in an information-poor economy the equilibrium is efficient, there is wasteful competition for scarce attention if the economy is information-rich. The paper shows that efficiency can be implemented by a linear tax on attention seeking activities.

The analysis starts from the basic premise of behavioral economics that economic modelling must account for psychological facts - when discussing economic decision making, but also in equilibrium analysis and for macroeconomic or policy questions. The paper is based on a fundamental restriction pointed out by attention and perception psychology: Any behavior - whatever the degree of rationality of subjects - is contingent on a perceptual filter. This filter or "gate" imposes on individual behavior as well as on market interactions a constraint - in addition to the conventional budget and resource constraints. Media are an obvious example for gating. Inevitably, they present information in an order. And people pay attention to salient places, for instance to headlines or to the first items displayed by the search engine in response to a query. The paper 
builds on attention psychology and Weber's law of Psychophysics for the foundation of consumer behavior that takes gating constraints into account.

Experience of limited attention and information stress is familiar from everyday life. Attention psychology distinguishes between endogenous (or voluntary) and exogenous (or non voluntary/automatic) control. In Kahneman's [1973] words: Voluntary attention means that "the subject attends to stimuli because they are relevant to a task that he has chosen to perform" (p. 4), whereas involuntary attention is related to the level of arousal which "is largely controlled by the properties of the stimuli to which the organism is exposed " (p. 3). The former is also addressed as "top-down control" of attention while the latter is called "bottom-up processing". Both are important: "Channel selection is guided by top-down influences (e.g. current goals) as well as bottom-up influences (e.g. stimulus intensity)" (Lachter, Forster and Ruthruff [in Press], p. 2). In recent years, several economists have taken up the problem of limited attention and attention seeking ${ }^{1}$, which Camerer [2003] listed among the important topics for behavioral economics. These studies shed light on the aspect of goal-driven voluntary attention allocation. Gabaix and Laibson [2004] speak of directed cognition. The main focus is on the processing of a crowded agenda (receiver perspective): How do individuals allocate a given time budget on different sources of information (Gabaix, Laibson and Moloche [2003])? Which parts of macroeconomic data should rational agents evaluate if limited information-processing

\footnotetext{
${ }^{1}$ Also authors from other scientific fields have begun to study the consequences of limited attention. For instance, an account of the art of achieving attention by scientific publishing is given by Klamer and van Dalen [2002] . Davenport and Beck [2001] deal with attention from the perspective of business economics. Also Shapiro and Varian [1999] discuss business strategies for attracting attention. Hirshleifer, Lim and Teoh [2002], and Hirshleifer and Teoh [2003] analyze the implications of limited attention of investors for firms' information policy and the financial market equilibrium. Dukas [2004] reviews the evolutionary causes of limited attention and its role for the fitness of animals. And, the most salient proof of a new and severe problem of information congestion - the flood of spam - has induced computer scientists to pay attention to the "market for attention". One of the ideas is to implement such a market in a quite conventional way by requiring postage for E-mail. Kraut et al. [2002] did first laboratory experiments on the effectiveness of such proposals.
} 
capacity forces them to discard part of the data (Sims [2003])? How often do inattentive producers or consumers update their information for adjusting prices or savings plans (Reis [2004 a,b])?2 My contribution is complementary. It focusses on automatic attention control by stimulus strength. The active part is played by the attention seekers - the firms - rather than by the individuals paying attention. Goal-oriented information processing requires some prior knowledge on the information sources. ${ }^{3}$ Without prior knowledge, to focus on important rather than on irrelevant signals would require valuation of all signals in the first place. Limited attention means that this is impossible. In contrast, information content does not matter in the approach of this paper which focusses on attention control by stimulus intensity. In such an approach the interest shifts from the consumer to the supply side. How "strong" must an information source send to have impact (sender perspective)? How many different sources are perceived (i.e. survive) if all of them compete for attention by sending "look at me!" (equilibrium diversity)? The paper explains individually perceived diversity (local diversity), aggregate diversity (perceived by an outside observer or "global traveller") and the distinctiveness of the choice sets of different consumers.

The psychological fact of limited attention capacity does not automatically imply that attention is a scarce resource that restricts economic behavior. As Herbert Simon has pointed out, the new scarcity problem arises in an information-rich economy. Information "consumes the attention of its recipients. Hence a wealth of information creates a poverty of attention." (Simon [1971, p. 40]). One purpose of this paper is to explain scarcity of attention endogenously: Which changes in psychological and technological fundamentals turn an information-poor economy, in which only the economic resources

\footnotetext{
${ }^{2}$ Gabaix and Laibson [2002] show how the equity premium puzzle can be explained by limited investor attention.

${ }^{3}$ For instance, in the experiments of Gabaix, Laibson et al. [2004] subjects know that boxes farther to the left contain more information than boxes at the right side. In the context of time series, properties like frequency or serial correlation can provide guidelines for which signals should be tracked and which should be ignored.
} 
are scarce, into an information-rich economy, in which scarcity of attention complements economic scarcity? ${ }^{4}$ For a rigorous answer, a two-stage competitive equilibrium model is proposed. In a first stage, attention seeking agents compete for the attention of receivers by sending signals. At the second stage, the agents who get through the perceptual filter compete for the budget of receivers in a Dixit and Stiglitz [1977] monopolistic competition framework. There is no heterogeneity among senders or receivers and all outcomes are symmetric. The exogenous fundamentals are: The set of receivers (buyers) and psychological characteristics of their perception filter. The set of potential senders (sellers) with their range and cost structure for generating and distributing signals to the receivers. And the fundamentals of conventional economic competition (preferences, endowments, production technology). By determining the equilibrium we can identify the economic factors that are responsible for a switch from an information-poor to an information-rich economy: Technical progress in IT, rising income, international integration and powerful media channels. By comparing equilibrium results in an information-rich economy with the equilibrium outcomes in an information-poor world, we can also answer the question: What difference does the new scarcity problem make - not only at the individual level but for equilibrium predictions?

The paper is organized in the following way. Section 2 presents the psychological model of receiver behavior with limited attention. Section 3 describes the competition of sellers for the attention of buyers as well as their competition for the budget of attracted buyers. In Section 4, the equilibrium is characterized and an explicit criterion is established for determining whether an economy is information-rich or information-poor. Equilibrium

\footnotetext{
${ }^{4}$ Falkinger [2003] worked out the general mathematical structure of the interaction of massless senders and receivers, allowing for heterogeneity of agents. There however, scarcity of attention is imposed by assumption and the benefit of getting attention is exogenously given without modelling the perceptionconstrained economic competition. By contrast, this paper considers a more concrete psychological and economic structure to analyze competition for attention and conventional economic competition simultaneously and to determine endogenously whether an economy with finite information-processing capacities is information-rich or information-poor.
} 
diversity of items and firms, equilibrium strength of attention-seeking activities and the conventional economic equilibrium are derived for the information-poor and informationrich regime, respectively. In Section 5, the economic causes of scarcity of attention and its positive implications are shown by doing comparative-static equilibrium analysis. Section 6 deals with the normative aspects: Welfare, efficiency and new policy measures required in an information-rich economy. Section 7 considers the role of media-intermediated competition for attention. Section 8 analyses indirect psychological effects of intensified attention-seeking on the market power of firms, the sectoral structure of an economy and the consumption- and work-orientation of individuals. Section 9 summarizes the main results.

\section{The psychological model of limited attention}

Psychological research has identified two basic aspects of human information-processing: (i) The selection of the set of items to be processed according to a filter or gating mechanism, and (ii) the processing of the selected items by allocating limited mental resources on them. Pashler [1998] summarizes the empirical evidence of attention psychology under the term "controlled parallel processing" asserting "that capacity limits and perceptual gating both characterize human perceptual processing" (p. 224, emphasis Pashler). According to him, many psychological controversies result from the fact that some researchers identify the concept attention with the allocation of a limited processing capacity on a given set of items, while others relate attention to the selection of this set by a gating mechanism. The gating mechanism implies that the mental resources for informationprocessing are only allocated on those items which have passed the perceptual filter while the rest is ignored.

\subsection{Capacity allocation}

A good way to think about the problem faced by a sender (an information source), who addresses a receiver with some signal strength, is Kahneman's [1973] dual task approach. 
According to this approach, individuals are endowed with a certain mental capacity. The speed and quality of processing an additional signal depends on the "spare capacity" left after the load imposed by exposure to other signals. Denote by $\bar{h}$ the receiver's information-processing capacity and let $\tau$ be total signal exposure of the receiver. Symmetry requires that the capacity left per signal - denoted by $v$ - is given by the equation

$$
v \tau=\bar{h}
$$

Capacity $\bar{h}$ is not an invariably natural constant but depends on receiver effort, which typically rises when higher signal exposure raises the level of arousal. "However, the increase is inefficient to maintain performance at a constant level of speed and quality." More precisely this applies if load $\tau$ is above a certain level. "At low values of load, the response of the system is approximately linear, and there may be little or no interference between tasks" (Kahneman [1973, p. 16]). Pashler [1998, p. 162] summarizes the vast experimental evidence provided by attention psychology as follows: "... it appears that capacity limits do exist beyond a certain point; with stimulus load below this level, processing appears to be parallel and free of capacity limits." Formally, this can be modelled by specifying capacity as follows:

$$
\bar{h}(\tau)=\left\{\begin{array}{lll}
\tau & \text { if } & \tau<\tau_{0} \\
\tau^{\beta} \tau_{0}^{1-\beta} & \text { if } & \tau \geq \tau_{0}
\end{array}\right.
$$

where $\beta \in[0,1)$, and $\tau_{0} \geq 1$ is an exogenously given threshold. (Coefficient $\tau_{0}^{1-\beta}$ guarantees that $\bar{h}$ is continuous at $\tau_{0}$.)

Symmetry requires $v=\bar{h}(\tau) / \tau$. Thus, attention paid per signal by a receiver exposed to $\tau$ is given by the

$$
v= \begin{cases}1 & \text { if } \tau<\tau_{0} \\ \left(\tau_{0} / \tau\right)^{1-\beta} & \text { if } \tau \geq \tau_{0}\end{cases}
$$

Total signal exposure $\tau$ is the sum of signals sent by many attention seeking agents. It is assumed that single agents have zero mass, i.e. $\tau$ and $v$ are given for individual 
attention seekers. Thus, an agent sending with strength $\sigma$ attracts attention $\sigma v$. This attention determines the mental impact of the sender on the receiver. "Attended events are more likely to be perceived consciously, and more likely to be perceived in detail. They have a higher probability of eliciting and controlling responses, and they are more likely to be stored in permanent memory" (Kahneman [1973], p. 68). In sum, the impact of a source, which is seeking attention with signal strength $\sigma$, on the mind of a receiver, who spends attention $v$ per signal, is given by

$$
z=\sigma v .
$$

\subsection{Gate}

The question considered so far was: Given an information source entering a receiver's mind with strength $\sigma$, how much receiver attention is allocated on $\sigma$. The question now is: Which information sources enter the receiver's mind, i.e. pass the perceptual filter?

The psychological literature discusses two types of thresholds. First, even in a completely silent environment, a signal must have a certain absolute strength $\sigma_{0}$ to be perceived. I normalize $\sigma_{0}$ to unity. Thus, if $\tau<\tau_{0}$ so that there is no crowding, an information source passes the perceptual filter if and only if $\sigma \geq 1$. However, if $\tau \geq \tau_{0}$ there is also a relative threshold: A source must be distinguishable from other information sources competing for entry by sending signals. "Just noticeable differences" (JNDs) are a central concept of Psychophysics. According to Weber's law, there is a basic invariance namely "that in order for a change in a stimulus to become just noticeable, a fixed percentage must be added. In other words, what it takes to make a perceived difference is a relative matter" (Stevens [1986], p. 9). ${ }^{5}$ Formally, in order to be perceived as an

\footnotetext{
${ }^{5}$ Note that the more controversial law of Fechner on the subjective sensation caused by concatenating JNDs plays no role in the presented model of receiver behavior. The question is how many senders pass the perceptual filter, i.e. are identified as a separate information source, not what receivers feel or how they value signal exposure. (Beside Stevens [1986], see e.g. Luce [2004] on the controversal issue of how to measure those subjective sensations.)
} 
additional information source by a receiver exposed to $\tau \geq \tau_{0}$, a sender must send at least with strength $\sigma_{\min }=k \tau$, where $k$ is a constant. $k=1 / \tau_{0}$ guarantees $\sigma_{\min }=1$ at $\tau=\tau_{0}$. In sum we have the "gate"

$$
\sigma_{\min }=\left\{\begin{array}{lll}
1 & \text { if } & \tau<\tau_{0} \\
\tau / \tau_{0} & \text { if } & \tau \geq \tau_{0}
\end{array}\right.
$$

By sending $\sigma_{\min }$, an attention seeking agent acquires one "entry" on the mind of an addressed receiver.

\subsection{Media gate}

The psychological fundamentals of attention allocation and gating determine receiver behavior under direct sender-receiver interaction. However, in an information-rich world the interaction often involves media-intermediation. An attention seeking agent can reach receivers directly within some range $\rho$. For reaching a wider range, an agent's signals must be transmitted through information channels like search engines or mass media. Such channels necessarily present information in an order - for instance, as headline or small notice, at the front page or at some page further behind. Let $n$ denote the rank of an information-source in the media.

Psychological evidence shows that media consumers pay attention to salient news. A better rank means more salience. To make things simple, suppose that media consumers pay attention to the first $n_{0}$ items and neglect the rest. For instance, Internet searchers look at the first page of links shown by the search engine in response to a query. Then, an information source must achieve at least rank $n_{0}$ to get through the perceptual filter under media-intermediation. Suppose further that the ranking is fair in the sense that it is based on the impact of sources in the direct interaction between senders and receivers. According to (2.4), the impact of a source on a directly addressed receiver is $z=\sigma v$. Thus, an agent reaching $\rho$ receivers has direct impact $Z=\rho z=\rho \sigma v$. The higher score $Z$, the better the rank of the source. Let $\sigma_{n_{0}}$ be the strength of the source with marginal rank $n_{0}$. Then, condition $n \leq n_{0}$ for perception in the media is equivalent to $Z \geq \rho \sigma_{n_{0}} v$ 
or $\sigma \geq \sigma_{n_{0}}\left(\geq \sigma_{\min }\right)$. Hence, apart from the psychological gate, also media-gate requires from senders some minimal strength if they want to get an entry on the minds of a large range of receivers. The general economic implications of limited attention can be discussed without distinguishing whether the gating comes from psychological limitations or from media-intermediation. The additional insights from explicitly taking into account the role of media are discussed in Section 7.

\section{Economic competition under limited attention}

Agents seek attention since participation in economic competition requires perception by trading partners. Take for instance a firm. The better it succeeds to bring its projects to the attention of investors the more likely it attracts funds for realizing the projects. In a similar way, the more a firm is on the mind of consumers the higher are the sales it can expect. In terms of the sender-receiver model presented in Section 2, firms have to acquire "entries" on the minds of buyers.

Denote by $\mathrm{T}=[0, T]$, the set of firms. Suppose the available technology for distributing information is such that signals are diffused randomly across the economy and each firm $t \in \mathrm{T}$ can reach a mass $\rho$ of receivers. Let $R$ be the total mass of buyers. The technically feasible range $\rho$ may be smaller or larger than $R$ so that the effective range is $r \equiv \min \{\rho, R\}$. According to Section 2, by sending with strength $\sigma_{\min }$ a firm acquires one entry on the mind of each buyer in range $r$. Senders can be multiproduct firms by acquiring more than one entry. Let $q_{t}$ denote the number of entries acquired by $t \in \mathrm{T}$.

\subsection{Competition for attention}

Acquiring $q_{t}$ entries requires to generate and send a volume of $\sigma_{t}=q_{t} \sigma_{\min }$ signals, where $\sigma_{\mathrm{min}}$ is exogenous to the single firm. Let the cost of producing and distributing strength $\sigma$ over range $r$ be given by

$$
C(\sigma, r), \quad C_{\sigma}>0, C_{\sigma \sigma}>0, C_{r} \geq 0
$$


where subscripts denote partial derivatives. ${ }^{6}$ There is no spatial structure in the model. Cost $C$ may increase with the number of addressed receivers $r$, but does not depend on the distance between sender and receiver. (This is different from transportation costs.) Even $C_{r}=0$ is not implausible, since with modern information technologies like the Internet diffusion costs don't depend on how many receivers are addressed. The costs are incurred because being on buyers' mind is profitable. Each entry brings an item to the attention of a buyer. Let $\pi(q)$ be the profit that a firm can extract from a buyer through $q$ entries. $\pi(q)$ depends on the details of the market equilibrium realized, when a set T of sellers compete for the economic resources of a mass of $R$ subjects (see Section 3.3). In the consumer goods market, set $\mathrm{T}$ are the producers of which consumers are aware. In the financial market, $\mathrm{T}$ is the set of firms whose projects are competing for the funds of investors. In any case, there is some form of conventional economic interaction between the buyers and the sellers on their mind. For fixing ideas, monopolistic competition in the goods market is considered in Section 3.3.

Anticipating that $\pi\left(q_{t}\right)$ can be earned from each buyer addressed with strength $\sigma_{t}=$ $q_{t} \sigma_{\min }$ and having the capacity to reach $r$ receivers, each $t \in \mathrm{T}$ chooses

$$
\max _{q_{t}} \Pi\left(q_{t}\right) \equiv r \pi\left(q_{t}\right)-C\left(q_{t} \sigma_{\min }, r\right)
$$

taking $\sigma_{\min }$ as given.

\subsection{Perception constraint and diversity}

Buyers choose from the set of items that have passed the perceptual filter of the gating mechanism. Only items which are brought to the attention of a buyer with signal strength $\sigma_{\text {min }}$ have an entry on the buyers' mind and belong thus to their choice set. Let the set of items perceived by buyer $i$ be indexed by $\mathbf{M}_{i}$ and let $M_{i}$ denote the size of $\mathbf{M}_{i}$.

\footnotetext{
${ }^{6}$ Instead of assuming that distribution costs depend on the effective range $r$ we might assume that $C(\sigma, \cdot)$ is determined exclusively by technology, i.e. is a function of $\rho$ rather than $r$. The only consequence would be that $\sigma^{*}$ and $\kappa$ determined in Lemma 1 are functions of $\rho$ instead of $r$. Anyhow, this makes only a difference if $\rho>R$.
} 
According to the analysis in Section 3.1, each $t \in \mathrm{T}$ brings $q_{t}$ items to the attention of $r$ buyers drawn randomly from a total mass $R$. Hence, the size of an individual buyer's choice set is given by the equation

$$
M_{i}=\frac{1}{R} \int_{\mathrm{T}} r q_{t} d t \equiv M .
$$

It should be noticed that the choice sets $M_{i}$ of different buyers may overlap but generally are not identical (despite equal size). Only if each firm reaches all buyers, i.e. if $r=R$, all buyers are aware of the same items. I call size $M$ the local diversity. It measures of how many items an individual buyer is aware.

The fact that each item in $M_{i}$ is brought to the attention of the buyer with strength $\sigma_{\min }$ implies for the buyer's total signal exposure: $\tau=M \sigma_{\min }$. Substituting this for $\tau$ into (2.5), we obtain the following constraint on the economy: Either

$$
M=\tau<\tau_{0} \text { and } \sigma_{\min }=1
$$

or

$$
M=\tau_{0} \text { and } \sigma_{\min } \geq 1
$$

In the first case, we have an information-poor economy. Only the absolute perception threshold $\sigma_{\min }=1$ must be passed to be in a buyer's choice set. Its diversity, $M$, is determined by the competition of sellers for buyers. Section 4 will characterize the equilibrium diversity. In the second case, we have an information-rich economy. The size of a buyer's choice set is constrained by her or his limited perceptual capacity (represented by parameter $\tau_{0}$ ). The competition of sellers for being in the limited set of perceived items drives up $\tau$ and $\sigma_{\min }$ (see Section 4 for the determination of equilibrium signal strength).

At the aggregate level, the set of items perceived in the economy is given by the union $\mathrm{S}=\bigcup \mathrm{M}_{i}$ of individual choice sets. Let $S$ denote the size of $\mathrm{S}$. I call $S$ the aggregate diversity. It measures the number of items an outside observer of the economy would 
count. $S$ is equal to the total number of items brought to the attention of buyers by firms, i.e.

$$
S=\int_{\top} q_{t} d t
$$

Comparing (3.6) with (3.3), we get

$$
\frac{S}{M}=\frac{R}{r}
$$

which is a measure for the overlap between the individual buyers' choice sets. We may call this measure "distinctiveness". ${ }^{7}$ In the limit, if information technology allows distribution of signals within small neighborhoods, $S / M$ approaches infinity. In contrast, if each firm can reach the whole population, we have $S / M=1$.

\subsection{Competition for money}

The items which succeed to be attended by buyers compete for the buyers' budget in a conventional way. Since firms had to incur cost $C(\sigma, r)$ for attracting attention, there is imperfect competition in which firms earn the profits to cover $C(\sigma, r)$. I use the Dixit and Stiglitz [1977] model of monopolistic competition. This seems appropriate in particular for the goal to analyze the consequences of limited attention at a macroeconomic level.

Each buyer $i$ is endowed with budget $y$ and has CES preferences over the set of items $\mathrm{M}_{i}(\mathrm{~s})$ he is aware of:

$$
U=\left[\int_{M_{i}} x_{s}^{\varphi} d s\right]^{1 / \varphi}, \quad 0<\varphi<1
$$

This implies for each item the demand function

$$
x_{s}=\frac{y}{P_{i}} p_{s}^{-\varepsilon}, \quad s \in \mathbf{M}_{i}
$$

\footnotetext{
${ }^{7}$ Caplan and Cowen [2004] distinguish between "diversity as a menu of choice" and "diversity of cultural distinctiveness". Local diversity measures the first aspect while the second aspect is related to $S / M$.
} 
where $\varepsilon \equiv \frac{1}{1-\varphi}>1$ is the elasticity of substitution between items and $P_{i} \equiv \int_{\mathrm{M}_{i}} p_{s}^{1-\varepsilon} d s$ is a price index. A seller $t \in \mathrm{T}$ who has acquired $q_{t}$ entries on the buyer is a multiproduct monopolist for $q_{t}$ variants with independent demand $\left(\partial x_{s} / \partial p_{s^{\prime}}=0, s^{\prime} \neq s\right)$. Thus, firms sell all variants at the familiar monopoly price $p_{s}=p=\frac{\varepsilon c}{\varepsilon-1}$, where $c$ denotes unit production cost. Since prices are symmetric, a receiver buys $x_{s}=x=\frac{y}{M} \frac{\varepsilon-1}{\varepsilon c}$ of each variant. Firm $t$ extracts thus operating profits

$$
\pi\left(q_{t}\right)=q_{t}(p-c) x=q_{t} \frac{y}{\varepsilon M}
$$

from each of the $r$ buyers reached by $t$ 's signals. This is the profit anticipated in decision (3.2) about the strength with which a firm competes for attention.

Substituting (3.10) for $\pi\left(q_{t}\right)$ in (3.2), we can solve for $q^{*}=\arg \max \Pi(q)$. Given range $r$ and cost structure $C$ of the information technology, each active firm promotes $q_{t}=q^{*}$ variants, where $q^{*}$ depends on the required minimal signal strength $\sigma_{\text {min }}$ and on the conditions in the goods market as reflected in the term $\frac{y}{\varepsilon M}$.

Pay-off function $\pi(q)$ has form (3.10) in any competitive environment in which buyers spend a certain budget $y$ on the set of perceived items uniformly (e.g. investors who diversify their portfolio over random projects brought to their attention). Moreover, it should be noticed that strength $\sigma_{\text {min }}$ with which an item is brought to a buyer's attention does not enter directly in (3.10), only through the firm's choice of $q_{t}$. Also the buyer's attention level $v$ or the psychological impact of an item $\left(z_{0}=v \sigma_{\min }\right)$ does not matter. For the purpose of extracting money, only budget $y$ and elasticity $\varepsilon$ are relevant buyer characteristics. The levels of $\sigma_{\min }, v$ or $z_{0}$ may affect the buyer's subjective well-being. For instance, buyers may be stressed if signal exposure is high. But their demand behavior is determined by $y$ and $\varepsilon$. Section 8 extends the analysis and considers possible channels through which $y$ or $\varepsilon$ may be affected by psychological factors like $z_{0}$. This will not change the form of the demand or profit functions so that the presented analysis of firm behavior remains valid. For the single firm, $y$ and $\varepsilon$ are given anyway. Also the consequences of the gating mechanism for local and aggregate diversity are unaffected. Only the equilibrium 
values, derived in the next section for exogenous values of $y$ and $\varepsilon$, will change.

\section{Equilibrium analysis}

The model has six variables: $\sigma_{\min }, \tau, M, S, T$ and $q^{*}$. The first four variables describe the filter of perception (minimal signal strength for being perceived as separate item, total signal exposure, local and aggregate diversity of perceived items, respectively). Pair $T, q^{*}$ corresponds to the embedded economic competition - number of active firms and variants per firm.

$T$ and $q^{*}$ have to satisfy the familiar conditions for profit maximization and free entry:

$$
q^{*}=\arg \max \Pi(q) \text { and } \Pi\left(q^{*}\right)=0,
$$

where $\Pi$ is given by (3.2) and (3.10). Moreover, with $q_{t}=q^{*}$ the equations (3.3) and (3.6) reduce to

$$
M=\frac{r}{R} T q^{*} \text { and } S=T q^{*}
$$

As a consequence of limited attention, system (4.1) interacts with the perception constraint. A firm $t$ earns profit $\Pi\left(q^{*}\right)$ only upon the condition that the $q_{t}\left(=q^{*}\right)$ items of $t$ are perceived, that is, that $t$ occupies $q^{*}$ entries on receiver minds by sending to each entry with strength $\sigma_{\min }$. For the single sender, $\sigma_{\min }$ is an exogenous parameter, but in equilibrium it is endogenously determined. Depending on how intensively potential sellers grab for the attention of buyers, more or less strength is required to pass the perception gate. According to (3.4) and (3.5), two cases must be distinguished - the information-poor and the information-rich economy, respectively. In an information-poor economy, the buyers' signal exposure is low and they observe whatever sellers bring to their attention with strength $\sigma_{\min }=1$. Given this exogenously fixed threshold, firms enter with the optimal $q^{*}$ until profits are driven down to zero. $q^{*}$ together with $T$ determine diversity according to (4.2). In contrast, in an information-rich economy, the limited information-processing capacity of buyers constrains the number of variants they 
can perceive to $M=\tau_{0}$. Thus, if positive profits induce new senders to enter, they fail to attract attention or others are kicked out from the receivers' mind. Competition for attention drives up $\tau$ and $\sigma_{\mathrm{min}}$. This brings down profits. ${ }^{8}$ The following scheme (Figure 1) summarizes the structure of the model for the two regimes.

\section{Figure 1}

In the further analysis I determine whether an economy is information-poor or information-rich and characterize the equilibria in the two regimes. For a more specific discussion of the firms' costs of attracting attention it is useful to distinguish the cost of expanding the range of signal diffusion from the cost of signal production. I will illustrate the results for the cost structure

$$
C(\sigma, r)=\frac{1}{2} d \sigma^{2}+f(r)
$$

in which the two components are separated. $d$ is a positive constant representing the slope of the marginal cost of signal production. $f(r)$ (with $f^{\prime} \geq 0$ ) is the fixed cost of reaching $r=\min \{\rho, R\}$ receivers by the prevailing sender technology with range $\rho$.

The following lemma is useful for determining the impact of range $r=\min \{\rho, R\}$ on the equilibrium. (Proofs are provided in the appendix.)

Lemma 1 . For $C(\sigma, r)$ satisfying (3.1), let $\sigma^{*}(r)$ be the implicit function defined by $C\left(\sigma^{*}, r\right)=\sigma^{*} C_{\sigma}\left(\sigma^{*}, r\right)$ and define $\kappa(r) \equiv C_{\sigma}\left(\sigma^{*}(r), r\right)$ for later use. We have: (i) $d \sigma^{*} / d r \geq 0$ if $\sigma^{*} C_{r \sigma} \leq C_{r}$. (ii) For (4.3), $\sigma^{*}(r)=\sqrt{\frac{2 f(r)}{d}}, \kappa(r)=\sqrt{2 d f(r)}$. In equilibrium,

$$
q^{*} \sigma_{\min }=\sigma^{*}(r)
$$

The lemma establishes that range $r$ is a crucial determinant for how strong sellers send $\left(\sigma_{t}=q^{*} \sigma_{\min }\right)$ in competing for attention. Under plausible conditions regarding the

\footnotetext{
${ }^{8}$ According to $(3.2), \partial \Pi\left(q^{*}\right) / \partial \sigma_{\min }=-q^{*} C_{\sigma}<0$.
} 
costs of producing and distributing signals ${ }^{9}$, in particular under cost structure (4.3), the contest for attention induces firms to generate more signals when $r$ rises. ${ }^{10}$

As first main result I show which conditions are responsible for whether we have an information-rich or an information-poor economy. Only in the first case, attention is the scarce resource.

Proposition 1 (Scarcity regime). Let $\kappa(r)$ be the function defined in Lemma 1. An economy is information-rich (so that attention is a scarce resource) if

$$
r y / \varepsilon>\tau_{0} \kappa(r)
$$

If the inequality is reversed, the economy is information-poor (i.e. has no attention problem). If (4.5) holds with equality, the economy is information-rich by convention.

Corollary 1 . For cost function (4.3), condition (4.5) reduces to $r y>\tau_{0} \sqrt{2 d f(r)}$.

To my knowledge, this is the first attempt to characterize an information-rich economy, facing scarcity of attention, in terms of fundamentals. Conditions (4.5) and Corollary 1 clearly bring out why scarcity of attention has become an issue. When innovations allow to address a larger range of receivers (i.e. $r$ rises) without raising cost, or when the cost of sending signals decline, then an information-rich economy arises. Also if receivers get richer or less price sensitive, an information-rich economy becomes more likely since more attention-seeking sellers survive when larger profits $y / \varepsilon$ can be achieved.

11

The next proposition characterizes equilibrium firm number and signal exposure.

\footnotetext{
${ }^{9} \sigma C_{r \sigma}<C_{r}$ if the cost of expanding radiation range is not too sensitive to variations in the volume of transmitted signals. This is the case whenever setting up a diffusion capacity with a certain range $\rho$ involves mainly fixed costs, in particular when $C_{r \sigma}=0$.

${ }^{10}$ Under specification (4.3), $\sigma^{*}$ rises with $f$ (Property (ii)). The reason is that firms want to spread the higher fixed cost over more items and are thus competing more aggressively for attention.

${ }^{11}$ If $\rho<R$, population size $R$ plays no role for whether the economy is information-poor or informationrich. With $R$ rises not only $T$ but also aggregate absorption capacity $S$. Signal exposure per receiver, i.e. $\tau=\sigma_{\min } M$, remains unaffected (see Propositions 2, 3 and 4).
} 
Proposition 2 (Firm number and signal exposure). Let $\sigma^{*}(r)$ and $\kappa(r)$ be the functions defined in Lemma 1. In equilibrium: $T=\frac{R y}{\varepsilon C\left(\sigma^{*}(r), r\right)}$ and $\tau=\frac{r y}{\varepsilon \kappa(r)}$. For cost function (4.3): $T=\frac{R y / \varepsilon}{2 f(r)}$ and $\tau=\frac{r y / \varepsilon}{\sqrt{2 d f(r)}}$.

Irrespective of the scarcity regime, more firms are active if the cost of bringing their products to the attention of buyers can be covered by larger $(R)$ or more profitable $(y / \varepsilon)$ markets. The firm number does not depend on $\tau_{0}$. Although there is no direct relationship to the scarcity regime, equilibrium firm number and scarcity regime are correlated since both the equilibrium value of $T$ and criterion (4.5) depend on $y / \varepsilon$ and in general also on $r .{ }^{12}$ Since signal exposure is essentially determined by the number of firms and the signal strength per firm $\left(\sigma^{*}(r)\right)$, also $\tau$ does not directly depend on the scarcity regime. But it always increases with sender range and market size - the same factors which move an economy from the information-poor to the information-rich regime. All other variables depend on the scarcity regime.

The next proposition characterizes the equilibrium in an information-poor economy.

Proposition 3 (Information poor economy). Let $\sigma^{*}(r)$ and $\kappa(r)$ be the functions defined in Lemma 1. In the equilibrium of an information poor economy: $\sigma_{\min }=1, q^{*}=\sigma^{*}(r)$, $S=\frac{R y}{\varepsilon \kappa(r)}, \tau=M=\frac{r y}{\varepsilon \kappa(r)}$.

Corollary 3 . For cost function (4.3), the equilibrium values in an information-poor economy are: $\sigma_{\min }=1, q^{*}=\sqrt{\frac{2 f(r)}{d}}, S=\frac{R y / \varepsilon}{\sqrt{2 d f(r)}}$ and $\tau=M=\frac{r y / \varepsilon}{\sqrt{2 d f(r)}}$.

In an information-poor economy, total signal exposure is so low that each source sending with strength 1 is perceived regardless of what competitors do. Nonetheless, a firm that wants to sell an item has to incur the cost of producing and distributing $\sigma_{\min }=1$ to get for the item an entry on buyer minds. This limits the number of entries that a firm wants to acquire as well as the number of firms surviving in equilibrium. As

\footnotetext{
${ }^{12}$ Note however that $T$ may be invariant with respect to variations of range $r$, namely if $f^{\prime}(r)=0$, whereas for (4.5) $r$ always plays a direct role even if not relevant in the cost structure.
} 
a consequence, also the number of supplied items is limited. In sum, the scarce supply of items - not the limited capacity of attention - determines the diversity perceived locally or in the aggregate. As Proposition 3 and its Corollary show, $S$ increases with the size of the economy $(R y)$ and decreases with the cost of information. Local diversity increases with $r / \kappa(r)$, in particular, as long as $\rho<R$ (so that $r=\rho$ ), if technical progress allows wider diffusion of signals without raising costs too much. This corresponds to conventional economic thinking. More precisely, an increase in $r$ increases $M$ if $r \kappa^{\prime}(r) / \kappa<1$, i.e. if the cost of generating and distributing signals is inelastic with respect to $r$. For cost structure (4.3), this condition reduces to $r f^{\prime}(r) / f(r)<2$, since $\kappa(r)=\sqrt{2 d f(r)}$. Thus, the essential point guaranteeing that $r / \kappa(r)$ rises with $r$ is that fixed cost $f$ does not react too sensitively to $r$. In the following this is often tacitly assumed when effects of increasing range are discussed informally.

In an information-rich economy the situation is reversed in some sense. The diversity of perceived items is determined by the limited capacity of attention. And changes in market size, radiation range or cost affect the intensity of competition, in particular the strength with which sellers compete for attention.

Proposition 4 (Information-rich economy). Let $\sigma^{*}(r)$ and $\kappa(r)$ be the functions defined in Lemma 1. In the equilibrium of an information-rich economy: $\sigma_{\min }=\frac{r y}{\tau_{0 \varepsilon \kappa(r)}}, \quad q^{*}=$ $\frac{\tau_{0} \varepsilon C\left(\sigma^{*}(r), r\right)}{r y}, \quad S=\tau_{0} R / r, \quad M=\tau_{0}$ and $\tau=\frac{r y}{\varepsilon \kappa(r)}$.

Corollary 4. For cost function (4.3), the equilibrium values in an information-rich economy are: $\sigma_{\min }=\frac{1}{\tau_{0}} \frac{r y / \varepsilon}{\sqrt{2 d f(r)}}, q^{*}=\tau_{0} \frac{2 \varepsilon f(r)}{r y}, S=\tau_{0} R / r, M=\tau_{0}$ and $\tau=\frac{r y / \varepsilon}{\sqrt{2 d f(r)}}$.

In the information-rich equilibrium attention capacity is fully exhausted. As a consequence, the diversity of items perceived by a single individual, $M$, is determined by this capacity - not by the scarce supply of items. According to Proposition 2, if the costs of sending signals on offered items decline or if a larger or richer population of buyers can be reached, the number of sellers $T$ grabbing for attention increases. According to 
Proposition 4 and its Corollary, also $\sigma_{\min }$ rises, that is, they are grabbing more strongly. Since attention capacities are exhausted, this means at the same time less perceived items per seller, i.e. a lower $q^{*}$. Note that for $\sigma_{\min }$ and $q^{*}$, firm range $r$ rather than $R$ is important. They don't coincide if $\rho<R$. In this case, an attention seeking agent has no motive to change behavior in reaction to an increased population of buyers, since any way only $\rho(<R)$ can be reached. For aggregate diversity, $S$, only population size $R$ relative to range $\rho$ matters besides attention capacity. The fundamental difference between an information-poor and an information-rich economy with respect to diversity is: In both regimes an increase of $\rho(<R)$ brings local diversity closer to aggregate diversity. But in an economy with scarcity of attention this happens through a decline in aggregate diversity - not by a rise in local diversity which has already reached the limit consistent with attention capacity parameter $\tau_{0}$.

\section{Causes and consequences of an information-rich economy}

\subsection{Causes}

Which changes are responsible for the emergence of scarcity of attention as a "new scarcity problem"? Proposition 1 gives a precise answer in terms of economic fundamentals: Excluding the ad hoc explanation that people's processing capacity $\tau_{0}$ has declined, the economic attractiveness of being on people's mind must have increased or the cost of being there has declined. According to (4.5), an economy is

$$
\begin{aligned}
& \text { IP if } \frac{r}{\kappa(r)} \frac{y}{\varepsilon}<\tau_{0}, \\
& \text { IR if } \frac{r}{\kappa(r)} \frac{y}{\varepsilon}>\tau_{0},
\end{aligned}
$$

where IP stands for information-poor and IR for information-rich. The first component, $r / \kappa(r)$, describes the available possibilities of attention seeking: $\kappa(r)$ is the equilibrium marginal cost of producing and distributing signals, $r$ is the range of buyers that can be 
reached. The second component, $y / \varepsilon$, reflects the attractiveness of attention seeking: $y$ is the representative buyer's budget and $\frac{1}{\varepsilon}\left(=\frac{p-c}{c}\right)$ is the profit margin. Figure 2 illustrates how the interaction of cost and revenue components determines whether we are in an information-poor or in an information-rich economy.

\section{Figure 2}

On the one side, rising per-capita income or an increase in the degree of monopoly power move the economy upward towards the frontier of information-richness (and thus scarcity of attention). On the other side, a rise in radiation range or declining cost of radiation also move the economy towards the frontier. The interaction of the two sides is important. Whereas in economy A with rich buyers and/or high degree of monopoly the shown progress in radiation possibilities induces a regime shift from IP to IR, the same progress does not turn economy B (with lower buyer budgets or lower degree of monopoly) into an information-rich economy. (In an analogous way, the effects of changes in $y$ or $\varepsilon$ depend on the economy's initial position.)

With respect to the change in radiation possibilities, two cases must be distinguished. Since $r=\min \{\rho, R\}$, we have $r / \kappa(r)=\rho / \kappa(\rho)$ as long as $\rho<R$. In this case, progress in information technology (for instance, new media channels) is the relevant cause for making an economy information-rich. IT-progress may allow firms to address their (given range of) buyers at a lower cost or gives them access to a wider range of buyers without increasing information cost too much.

If $\rho \geq R$, the range of the existing information-technology is so powerful that the whole population can be reached. In this case, range-increasing technical progress has no effect. Only access to a larger or richer mass of buyers, for instance foreign buyers by international integration, changes the opportunities for attention-seeking. Suppose there is a foreign population $R_{F}$ with income $y_{F}$, whereas home population is $R_{H}$ with income $y_{H}$. If $\max \left\{R_{H}, R_{F}\right\}<\rho \leq R_{H}+R_{F}$, foreign buyers can be reached with the existing technology of range $\rho$. Assuming that the cost of information distribution does 
only depend on the used technology, we have $\bar{\kappa}=\kappa(\rho)$ regardless of whether $\rho$ can be fully utilized (by addressing foreign buyers) or not (in the closed economy). Thus, we have in autarky

$$
\tau_{k}=\frac{R_{k} y_{k}}{\bar{\kappa} \varepsilon}, \quad k=H, F
$$

and under international integration

$$
\tau_{I}=\frac{\min \left\{\rho, R_{H}+R_{F}\right\} y_{w}}{\bar{\kappa} \varepsilon}
$$

where $y_{w}=\frac{R_{H} y_{H}+R_{F} y_{F}}{R_{H}+R_{F}}$ is the income of the representative agent of the integrated world. ${ }^{13}$ If $y_{w} \geq y_{k}$, a switch from (5.2) to (5.3), shifts the economy outwards in Figure 2. The possibility to attract foreign buyers increases the incentives for attention seeking, which increases signal exposure. Whether or not this shifts an economy across the frontier into the information-rich regime depends on size and richness of the other country. For example, if in autarky the economy is at point $\mathrm{B}$, then integration with a small foreign economy leads to $B_{1}$ which is still information-poor. However, integration with a larger economy shifts the economy to some point $B_{2}$, turning thus an information-poor economy without attention scarcity into an information-rich economy with scarcity of attention.

\subsection{Consequences}

The effects of changes in the economic fundamentals on the equilibrium were characterized in Proposition 2 to 4. As discussed in the interpretation of Proposition 2, there is no regime switch with regard to equilibrium firm number and signal exposure. Equilibrium signal exposure $\tau=\frac{r y}{\kappa(r) \varepsilon}$ summarizes the interaction of the exogenous factors relevant for the scarcity regime according to (5.1). Therefore, it is useful to discuss the effects of a switch from IP to IR on other variables by looking on how they vary with $\tau=\frac{r y}{\kappa(r) \varepsilon}$. Figure 3 shows the effects for the case that $\rho \leq R$ (i.e. $r=\rho$ ).

\section{Figure 3}

\footnotetext{
${ }^{13}$ Note that there are no distant-dependent transportation cost in the model so that firm location does not matter.
} 
As long as an economy is information poor, an increase in the incentives for attention seeking is fully transmitted into increased diversity for the individual buyers. In contrast, in the information-rich economy they intensify the competition for attention and raise the signal strength required for an item to be noticed by the buyers. The effects on aggregate diversity depend on where the increase in signal exposure comes from. If technical progress lowers signal cost $\kappa$ (leaving feasible radiation range $\rho$ constant), then aggregate diversity rises in an information-poor economy and remains constant in an informationrich economy. In contrast, if $\rho$ rises while $\kappa$ does not change, then $S$ remains constant in IP and declines in IR. ${ }^{14}$ Cultural distinction $S / M(=R / \rho$, according to (3.7)) declines in both regimes.

For $\rho>R$, international integration was seen to be an important cause for increased attention seeking incentives. To see the consequences of international integration, consider two economies $k=H, F$ and suppose that technically the available information technology allows to cover the population of both countries together, i.e. $\min \left\{\rho, R_{H}+R_{F}\right\}=$ $R_{H}+R_{F}$. Only institutional constraints restrict radiation range to $r_{k}=R_{k}$ in the closed economies. Then according to (5.2) and (5.3), equilibrium signal exposure in the integrated economy is given by the sum

$$
\tau_{I}=\tau_{H}+\tau_{F},
$$

where $\tau_{k}=\frac{R_{k} y_{k}}{\bar{\kappa} \varepsilon}, k=H, F$, is equilibrium signal exposure in the closed economies. Thus, the inhabitants in an information-poor economy $H$ with $\tau_{H}<\tau_{0}$ experience a regime switch to an information-rich world (with scarcity of attention) if $H$ integrates with an economy $F$, in which $\tau_{F}>\tau_{0}-\tau_{H}$. (Obviously, also the integrated economy will remain information-poor, if $\tau_{F}<\tau_{0}-\tau_{H}$.) The following proposition summarizes the effects of international integration on diversity.

Proposition 5 (International integration and diversity). Let $M_{k}, S_{k}$ and $M_{I}, S_{I}$ denote local and aggregate diversity in the closed and integrated economy, respectively. If $\rho \geq$

\footnotetext{
${ }^{14}$ According to Proposition 3 and $4, S=\frac{R y}{\varepsilon \kappa(\rho)}$ in an information-poor economy and $S=\tau_{0} R / \rho$ in an information-rich economy, respectively.
} 
$R_{H}+R_{F}$ : (i) Integration with another economy leads to $S_{I}=M_{I}>S_{k}=M_{k}$ if $k$ was information-poor before integration and to $S_{I}=M_{I}=S_{k}=M_{k}$ if $k$ was informationrich. (ii) If two information poor economies integrate to an information-poor world, then $S_{I}=S_{H}+S_{F}$. In all other cases, $S_{I}<S_{H}+S_{F}$. (iii) In any case, $\frac{S_{k}}{M_{k}}=\frac{S_{I}}{M_{I}}<\frac{S_{H}+S_{F}}{M_{k}}$.

Part (i) confirms that the total number of items $(S)$ supplied in the integrated world can never be smaller than in a closed economy. Moreover, an individual never loses in terms of experienced diversity $M$ (and thus in terms of utility as analyzed in Section 6) if an economy is integrated with another economy. Inhabitants of an information-rich closed economy are unaffected, since scarcity of attention limits diversity. Inhabitants of an information-poor economy gain by opening up, since foreign information sources become available. However, according to Part (ii), global diversity as seen from an outside look at the world (by a world traveller) is usually lower in the integrated world than the aggregate sum of items realized in isolated economies. Only if the world remains information poor after integration, global diversity is unaffected by international integration. Finally, Part (iii) tells us that the distintiveness of individual choice sets $(S / M)$ in the integrated economy $\left(S_{I} / M_{I}\right)$ is identical to the distinctiveness observed within the closed economies $\left(S_{k} / M_{k}\right)$. However, distinctiveness observed from the perspective of a world traveller declines if international integration allows firms to fully exploit global radiation possibilities. $^{15}$

\footnotetext{
${ }^{15}$ All claims of Proposition 5 are made under the assumption that global coverage $\left(\rho \geq R_{H}+R_{F}\right)$ is technically feasible. If $\max \left\{R_{H}, R_{F}\right\}<\rho<R_{H}+R_{F}$, the consequences of integration are more complex. Essentially, we have the following modifications: If $\rho$ is close to $\max \left\{R_{H}, R_{F}\right\}$ and the two economies are relatively heterogenous with respect to income or population size, then integration of an information-poor with an information-rich economy may lead to an information-poor international economy. Moreover, aggregate diversity and distinctiveness may be raised by integration. And the inhabitants of an economy may lose in terms of individually perceived items if their economy is integrated with a relatively information-poor economy.
} 


\section{Normative analysis}

This section compares the welfare level achieved in the competitive equilibrium with the efficient solution and shows how efficiency can be implemented by policy.

\subsection{Welfare in competitive equilibrium}

Valuation of diversity is a deep and controversial issue. The following remarks focus on a narrow economic aspect. Even within this narrow aspect important caveats have to be kept in mind. First, the discussion is based on a representative agent framework. Second, any possible role for extracting rents from limited attention is ignored since only free entry equilibria with zero profits are considered.

The analysis of the acquisition of entries on consumer minds was based on the assumption that firms which succeed in being attended earn gross profits given by function (3.10). Monopolistic competition for buyers with CES preferences over the items they perceive was shown to be a micro foundation of (3.10). In this case we have a clear welfare criterion, the buyers' utility (3.8) achieved in equilibrium. Since $p_{s}=p=\frac{\varepsilon c}{\varepsilon-1}$ and

$x_{s}=x=\frac{y}{M} \frac{\varepsilon-1}{\varepsilon c}$ in equilibrium, we have $U=M^{\frac{1-\varphi}{\varphi}} \frac{y(\varepsilon-1)}{\varepsilon c}$ for the representative agent's utility level. Substituting $M=\frac{r y}{\varepsilon \kappa(r)}$ from Proposition 3, we have

$$
U^{P}=\left(\frac{r}{\kappa(r)}\right)^{\frac{1-\varphi}{\varphi}}\left(\frac{y}{\varepsilon}\right)^{\frac{1}{\varphi}} \frac{\varepsilon-1}{c},
$$

in an information-poor economy. And substituting $M=\tau_{0}$ from Proposition 4 , we obtain

$$
U^{R}=\tau_{0}^{\frac{1-\varphi}{\varphi}} \frac{y(\varepsilon-1)}{\varepsilon c}
$$

for the information-rich economy. Comparing $U^{R}$ with $U^{P}$, we get $U^{P}<U^{R}$ if and only if $\frac{y r}{\varepsilon \kappa(r)}<\tau_{0}$ which is exactly the condition for an information-poor economy. Hence,

$$
U^{P}<U^{R}
$$

To live in an information-rich world is definitely better than life in an information-poor world. As long as attention is no scarce resource, progress in information technologies 
(raising radiation range $\rho$ or lowering signal cost $\kappa$ ) or international integration (increasing $r$ if $\rho \geq R$ ) increase the diversity of items buyers are aware of. Thus utility increases until the economy is information-rich and attention is scarce. In the information-rich economy IT-progress or globalization have no further effect on $U$ (except possibly through $y, \varepsilon$ or $c$, see Section 8), since scarce attention limits the variety of perceived items.

With respect to the interaction of the effects of income and information parameter $r / \kappa(r)$ on utility, we have:

$$
\frac{\partial^{2} U^{P}}{\partial y \partial(r / \kappa)}>\frac{\partial^{2} U^{R}}{\partial y \partial(r / \kappa)}=0 .
$$

In an information-poor economy changes in the fundamentals of the information sector affect the marginal utility of income and thus, for instance, the incentives to work (see Section 8). In an information-rich economy the marginal utility income is independent of $r / \kappa . \partial U^{R} / \partial y$ can only be increased if production cost $c$ or mark up factor $\frac{\varepsilon}{\varepsilon-1}$ decline or if the individuals' mental capacity $\tau_{0}$ improves.

The above comparison is based on perceived diversity $M$. In an information-rich economy a further welfare issue arises which is beyond the presented model. In an informationpoor economy every agent who has an economically viable idea or product can participate in the competition for buyers, since there are free entries on the buyers' mind. In contrast, in the information-rich economy entries on receiver minds are exhausted. Since there are so many and powerful potential information sources, attention necessarily focusses on a subset of potential sources. Since content can only be evaluated after an item has passed the perceptual filter, the selection of this subset cannot be based on content. Therefore, only the measure of the subset in the focus of attention is determined in the considered equilibrium. For welfare, however, content obviously matters. For instance, I can read a certain number of pages (written with standard clarity). How much I benefit from the reading is a different issue. The problem is: In the morning I could have chosen a different set of pages (of equal size and clarity), but then I wouldn't have seen the other pages. Moreover, the most interesting pages may not be in the market. The presented analysis of 
limited attention as a scarce resource points out that economic competition is contingent on a perception filter and that the set of perceived items is a subset of the economically possible items. In an information-rich economy, there is no guarantee that the perceived items are the best possible items.

\subsection{Efficiency}

Also a social planner has to account for the behavioral facts of attention psychology, in particular the perceptual gate (2.5). Moreover, the financial restriction that earnings must cover production as well as information cost has to be satisfied. Suppose that the planner chooses to inform through $T$ senders about $S=q T$ items with signal strength $\sigma_{\text {min }}$ per item. Then, total information costs to be covered are

$$
K \equiv C\left(q \sigma_{\min }, r\right) T
$$

The costs can be financed by two instruments: Prices and taxes. Each item can be brought to the attention of $r$ buyers. If a buyer consumes $x$ units of each item that (s)he perceives, and if the price per unit is $p$, the earnings per item are $r(p-c) x$. Total earnings are given by

$$
E \equiv \operatorname{Sr}(p-c) x
$$

The difference $E-K$ has to be balanced by public funds $D=E-K$. If $D<0$, the social planner imposes on each of the $R$ individuals a lump-sum tax $D / R$. If $D>0$, a lump-sum transfer $D / R$ is paid to each individual. Thus, disposable individual income is given by

$$
y^{n}=y+(E-K) / R \text {. }
$$

A buyer with preferences (3.8) and budget $y^{n}$ purchases

$$
x=\frac{y^{n}}{p M}
$$


of each of the $M$ perceived items and achieves utility level

$$
U=M^{\frac{1-\varphi}{\varphi}} \frac{y^{n}}{p} .
$$

Proposition 6 (Planner solution). Let $\sigma^{*}(r)$ and $\kappa(r)$ be the functions defined in Lemma 1. A social planner maximizing the representative agent's utility chooses: (i) $\sigma_{\min }^{*}=1$, $q^{*}=\sigma^{*}(r)$. (ii) If $(1-\varphi) y r / \kappa(r)<\tau_{0}$, then optimal local diversity is given by $M^{*}=$ $(1-\varphi) y r / \kappa(r)$. (iii) If $(1-\varphi) y r / \kappa(r) \geq \tau_{0}$, then $M^{*}=\tau_{0}$. (iv) Real disposable income is given by $y^{n} / p=\left[y-\kappa(r) M^{*} / r\right] / c$ and the maximal utility level $U^{*}=M^{* \frac{1-\varphi}{\varphi}} y^{n} / p$ is invariant with respect to the chosen financial policy $(p, D)$. (Moreover, $S^{*}=M^{*} R / r$ and $T=S^{*} / \sigma^{*}(r)$.

Since $1-\varphi$ is the inverse of the elasticity of demand $\varepsilon$, we have $(1-\varphi) y=y / \varepsilon$. Hence, in an information-poor economy (with $y r<\tau_{0} \varepsilon \kappa(r)$ ), the competitive equilibrium, characterized by Proposition 3, is efficient. Signal strength, local and aggregate diversity, firm number and items per firm coincide with the planner solution. Moreover, $U^{P}=$ $U^{*}$. However, in an information-rich economy (with $y r>\tau_{0} \varepsilon \kappa(r)$ ), the competitive equilibrium is inefficient. According to Proposition 4, the values for $M$ and $S$ are the same as in the planner solution. But competition for attention leads to wasteful signal exposure implying $\sigma_{\min }>1$ instead of $\sigma_{\min }^{*}=1$. As a consequence, we have a welfare loss

$$
U^{*}-U^{R}=\tau_{0}^{\frac{1-\varphi}{\varphi}} \frac{y}{\varepsilon c}\left(1-\frac{\tau_{0}}{\tau}\right)
$$

where $\tau=\frac{r y}{\kappa(r) \varepsilon}>\tau_{0}$ is signal exposure in the competitive equilibrium. (In contrast, $\sigma_{\min }^{*}=1$ and $M^{*}=\tau_{0}$ imply $\tau^{*}=\tau_{0}$ for signal exposure in the planner solution.) Equation (6.10) shows that the welfare loss from competition for limited attention is the higher, the farther the economic fundamentals $y / \varepsilon$ and $r / \kappa(r)$ are beyond the frontier of information-richness shown in Figure 2.

\subsection{Implementation of efficient solution}

In an information-poor economy - with no scarcity of attention - efficiency is achieved under laissez-faire. Intervention is required in the information-rich economy. 
According to Proposition 6, the planner is free in the choice of financial policy. Thus, price $p=\frac{\varepsilon}{\varepsilon-1} c$, resulting under monopolistic competition, is consistent with efficiency. However, the high profit margin implied by monopoly prices induces firms to invest into socially wasteful attention seeking by increasing their signal strength. For achieving efficiency, the competition for attention has to be constrained. The resources saved by keeping signal strength at the efficient level can be distributed to the consumers. Alternatively, one may intervene in the product market to bring down profit margins and to lower thus the firms' incentives for attention seeking. The following proposition characterizes the policy mixes by which efficiency can be achieved as a decentralized equilibrium in an information-rich economy.

Proposition 7 (Optimal policy). Let $\kappa(r)$ be the function defined in Lemma 1. The efficient solution can be implemented as competitive equilibrium by imposing on the signal strength $\sigma_{t}$ of attention seeking firms a linear tax $B=B_{0} \sigma_{t}$ and distributing the revenue to buyers. The required tax rate is

$$
B_{0}=\frac{\vartheta r y / \tau_{0}-\kappa(r)}{1-\vartheta}
$$

where $\vartheta=\frac{p-c}{p}$ is the price-cost margin allowed in the product market. $(\vartheta=1 / \varepsilon$ in the case of no intervention in the product market.)

The marginal tax rate $B_{0}$ increases with profit margin $\vartheta$ and increases in the fundamentals which make attention seeking more attractive - a larger radiation range $r$, lower signal cost $\kappa$ or richer buyer budgets $y$. If the product market is left to monopolistic competition so that $\vartheta=1 / \varepsilon$, the tax required for efficiency can be written in the form $B_{0}=\left(\frac{\tau}{\tau_{0}}-1\right) \frac{\varepsilon \kappa(r)}{\varepsilon-1}$, where $\tau=\frac{r y}{\varepsilon \kappa(r)}$ is the signal exposure that would result under laissez-faire competition for attention (characterized by Proposition 4). As shown in Section 5.1, $\tau$ is a measure for the degree of information-richness and $\tau / \tau_{0}$ is an indicator for the scarcity of attention. (According to Proposition 4, the minimum signal strength required for an item to be perceived in an information-rich laissez-faire economy 
is $\left.\sigma_{\min }=\tau / \tau_{0}.\right)$ Hence a rise in the scarcity of attention requires a higher tax on attention seeking activities.

\section{$7 \quad$ Media-Intermediation}

Apart from the perceptual filter at the individual level, under media-intermediation attention at the collective level is limited by the media gate discussed in Section 2.3. Suppose that the range for direct sender-receiver interaction is $\rho<R$, whereas the range of the media channel is $R$, i.e. the whole population can be reached by a source which successfully acquires an attended media place. By assumption there are $n_{0}$ such places. Thus, the probability that a firm gets a place in the media is

$$
\alpha=\min \left\{\frac{n_{0}}{T}, 1\right\}
$$

where $T$ is the equilibrium measure of attention seeking agents. ${ }^{16}$ This implies for a seller's expected range

$$
\bar{r}=\min \left\{\rho+\frac{n_{0}}{T}(R-\rho), R\right\}
$$

A firm generating strength $\sigma_{t}=q_{t} \sigma_{\min }$ and distributing $\sigma_{t}$ to $\rho$ receivers has cost $C\left(\sigma_{t}, \rho\right)$, according to (3.1). Since it is perceived in the media ${ }^{17}$ with probability $\alpha$, it can expect to reach $\bar{r}$ buyers so that expected profits are

$$
E \Pi\left(q_{t}\right)=\bar{r} q_{t} \frac{y}{\varepsilon M}-C\left(q_{t} \sigma_{\min }, \rho\right)
$$

according to (3.2) and (3.10).

Deriving the equilibrium in an analogous way to the analysis in Section 4, we obtain the following characterization of information-poor and information-rich economies under media mediation.

\footnotetext{
${ }^{16}$ We consider ex ante symmetric firms so that all agents have equal incentives to get through the media gate.

${ }^{17}$ Note that for symmetric agents the impact of the marginal firm is equal to the impact of the representative firm. Thus, $\sigma_{n_{0}}=\sigma_{\min }$. Any agent getting through the perceptual filter $\sigma_{\min }$ in direct interaction has the same chance to pass the media gate as well.
} 
Proposition 8 (Equilibrium under media-intermediation). For $\rho \leq R$, let $\sigma^{*}(\rho), \kappa(\rho)$ by the functions defined in Lemma 1. If direct radiation range $\rho$ is complemented by a fair media channel with range $R$ and gate capacity $n_{0}$, then, in equilibrium

$$
\bar{r}=\min \left\{\rho+\frac{n_{0} \varepsilon}{R y}(R-\rho) C\left(\sigma^{*}(\rho), \rho\right), R\right\},
$$

and $\tau=\frac{\bar{r} y}{\varepsilon \kappa(\rho)}, T=\frac{R y}{\varepsilon C\left(\sigma^{*}(\rho), \rho\right)}$. Moreover: (i) If $\frac{\bar{r} y}{\varepsilon \kappa(\rho)}<\tau_{0}$, the economy is information-poor and only $M=\frac{\bar{r} y}{\varepsilon \kappa(\rho)}$ depends on the media, whereas $\sigma_{\min }=1, q^{*}=\sigma^{*}(\rho)$ and $S=\frac{R y}{\varepsilon \kappa(\rho)}$ are determined by direct range $\rho$. ii) If $\frac{\bar{r} y}{\varepsilon \kappa(\rho)}>\tau_{0}$, the economy is information-rich with $M=\tau_{0}, \sigma_{\min }=\frac{\bar{r} y}{\tau_{0} \varepsilon \kappa(\rho)}, q^{*}=\frac{\tau_{0} \varepsilon C\left(\sigma^{*}(\rho), \rho\right)}{\bar{r} y}$ and $S=\tau_{0} R / \bar{r}$.

Equation (7.4) reflects the power of the media channel in magnifying the number of buyers that can be reached. ${ }^{18}$ No media-intermediation means that $n_{0}=0 . \rho=R$ means that media-intermediation plays no role since firms anyway can reach the whole population directly. In both cases, the results in Proposition 8 coincide with those in Section 4 . With $(R-\rho)>0$ the media channel allows to distribute information to a wider range, increasing thus the signal exposure of receivers and demanding more attention. However, attention seeking agents cannot be sure to reach the receivers in this way. The chance of getting through the media rises if high signal costs $C\left(\sigma^{*}(\rho), \rho\right)$, small markets or a low degree of monopoly (increasing $\frac{\varepsilon}{R y}$ ) decrease the incentives to seek attention.

Signal exposure rises with $\bar{r}$ so that the condition for an information-rich regime with scarcity of attention is satisfied more likely if media can be used for attention seeking other things being equal. If the economy remains information-poor nonetheless, the only effect of media-intermediation is a rise in the individually perceived diversity of items (Part (i) of the proposition). The reason is that attention capacity is no bottleneck as long as we have an information-poor regime. Therefore, there is room for an increase in the local diversity perceived by a single receivers. And the media bring items to the attention of buyers beyond direct range $\rho$. In an information-rich economy, mediaintermediated competition for attention has different effects. The media channel increases

\footnotetext{
${ }^{18}$ Note that for $\rho<R, \bar{r}<R$ if and only if $n_{0} C\left(\sigma^{*}(\rho), \rho\right)<R y / \varepsilon$.
} 
the range of an average information-source getting through the perceptual filter from $\rho$ to $\bar{r}$, given by (7.4). Since in an information-rich economy the limited individual attention capacity is binding, the equilibrium number of items perceived by a single receiver is not influenced by the characteristics of the media channel. However, since agents can expect to reach a wider-range of receivers, they intensify their effort to attract receivers and signal exposure of receivers rises as more signals are distributed over a wider range. As a result, minimum signal strength $\sigma_{\min }$ required for entering receiver minds rises and less entries $q^{*}$ are acquired per firm. Moreover, since all receivers are covered by the media channel there is more overlap between the items perceived by different receivers. Therefore, in an information-rich economy with $\rho<R$ aggregate equilibrium diversity $S$ is lower under media-mediation than when there is no media channel (i.e. if $n_{0}=0$ ). Note however that local as well as aggregate diversity always rise (from $M=\frac{\bar{r} y}{\varepsilon \kappa(\rho)}<\tau_{0}$ to $M=\tau_{0}$ and from $S=\frac{R y}{\varepsilon \kappa(\rho)}<\frac{R}{\bar{r}} \tau_{0}$ to $S=\frac{R}{\bar{r}} \tau_{0}$, respectively) if media mediation shifts an economy from an information-poor into an information-rich world. Cultural distinction, given by $S / M=R / \bar{r}$, declines with $\bar{r}$ regardless of whether the economy is information-poor or information-rich. Under media mediation more individuals perceive the same information sources.

Firms, although identical ex ante, are heterogeneous ex post. Those being lucky and getting attention through the media achieve positive profits $\Pi^{R}=R q^{*} \frac{y}{\varepsilon M}-C\left(\sigma^{*}(\rho), \rho\right)=$ $(R-\bar{r}) \frac{q^{*} y}{\varepsilon M}+E \Pi\left(q^{*}\right)>0$ since $E \Pi\left(q^{*}\right)=0$ in equilibrium. The others reach only $\rho<R$ buyers and suffer a loss $\Pi^{\rho}=(\rho-\bar{r}) \frac{q^{*} y}{\varepsilon M}<0$, since $\rho<\bar{r}$. Note that the decisions on the strength of attention seeking are made before firms are picked by the media. Attention in the media requires attention in direct interaction first, and under fair media agents who are equally attended in direct interaction have an equal chance to get media attention. Therefore, the costs $C\left(\sigma^{*}(\rho), \rho\right)$, incurred in the competition for attention, are sunk at the stage of competition for money. Reducing $q_{t}$ and thus $\sigma_{t}=q_{t} \sigma_{\min }$ ex post would lower the firm's revenue to $q_{t} \frac{\rho y}{\varepsilon M}<q^{*} \frac{\rho y}{\varepsilon M}$ and increase loss $\Pi^{\rho}$ even further. Substituting the equilibrium values from Proposition 8 for $q^{*}$ and $M$, we can rewrite the expressions for 
$\Pi^{R}$ and $\Pi^{\rho}$ to obtain

$$
\Delta \Pi \equiv \Pi^{R}-\Pi^{\rho}=\frac{R-\rho}{\bar{r}} C\left(\sigma^{*}(\rho), \rho\right),
$$

where $\sigma^{*}(\rho) \kappa(\rho)=C\left(\sigma^{*}(\rho), \rho\right)$ has been used. Substituting (7.4) for $\bar{r}$, we see that the profit risk increases with $C\left(\sigma^{*}(\rho), \rho\right)$ and $\frac{R-\rho}{\rho}$, i.e. if radiation cost are high and the range that can be reached in direct interaction is low compared to the media range. ${ }^{19}$ It decreases with the number of media slots per total buyer budget, i.e. if $\frac{n_{0}}{R y}$ rises. Finally, $\Delta \Pi$ is high if $\varepsilon$ is low so that the degree of monopoly power is high. The reason is that more firms are competing for attention in this case, which lowers $\bar{r}$ (see 7.4) and raises thus the advantage of those who reach the whole population by getting media attention.

\section{On interactions between psychological impact and economic fundamentals}

The analysis so far focussed on the following basic interaction between scarcity of attention and economic scarcity: The allocation of given budgets according to individual preferences is restricted to the items which pass the perceptual filter and enter the buyers' minds. The crucial psychological factor in this interaction is the gating mechanism, formally represented by minimal signal strength $\sigma_{\text {min }}$. The question how deeply an item enters the buyer's mind, i.e. psychological impact $z$, played no role for the determination of the equilibrium. (The gating mechanism only separates the items which enter from those which don't.) In other words, $z$ is a byproduct of competition for attention and money. According to $(2.3)$ - (2.5), the psychological impact $z_{0}=\sigma_{\min } v$ of an item which succeeds to be just distinguished by a receiver is given by

$$
z_{0}= \begin{cases}1 & \text { if } \tau<\tau_{0}, \\ \left(\tau / \tau_{0}\right)^{\beta} & \text { if } \tau \geq \tau_{0} .\end{cases}
$$

Parameter $\beta \in[0,1)$ is the elasticity with which a receiver can expand her or his attention capacity in reaction to stimulus exposure. For $\beta>0$, the psychological impact

\footnotetext{
${ }^{19}$ The expression for $\Delta \Pi$ becomes $\frac{1}{\frac{\rho}{(R-\rho)} C\left(\sigma^{*}(\rho), \rho\right)+\frac{n_{0} \varepsilon}{R y}}$.
} 
of an item increases with signal exposure in an information-rich economy. Due to scarcity of attention, the number of perceived items is limited but each item is brought to the receivers' attention with higher strength $\sigma_{\min }$.

The further analysis examines in how far our results change if impact $z$ is not only a mental byproduct of attention seeking but feeds back into buyer behavior. Two possible channels for such feedback effects are considered: $z_{0}$ may effect the price elasticity of demand (Section 8.1) or the budget $y$ they spend (Sections 8.2 and 8.3).

\subsection{Psychological effects of attention seeking on the degree of monopoly}

In an information-rich economy scarcity of attention limits the number of perceived items in the choice set of an individual to $M=\tau_{0}$. If changes in the economic fundamentals like progress in information technologies or larger markets raise the incentives for attention seeking, firms increase their signal strength. As a consequence, each perceived item enters the buyer's mind with higher signal strength. If $\beta=0$, this has no effect on the mental impact of an item. However, if $\beta>0$, additional mental effort is elicited and more attention is paid to each item. This may change the subjective valuation of the items. Assuming that preferences remain of type (3.8), attributing more importance to the single items in the choice set means that the elasticity of substitution $\varepsilon$ between the items declines and the marginal willingness to pay for an item rises. We can capture such an effect by assuming that elasticity $\varepsilon$ is a function of psychological impact $z_{0}$ :

$$
\widetilde{\varepsilon}\left(z_{0}\right)=\varepsilon z_{0}^{-\eta}, \quad 0 \leq \eta<1 / \beta,
$$

where parameter $\eta$ expresses the extent to which this psychological channel is effective. (For an equilibrium, the effect must be bounded so that $\beta \eta<1$ is guaranteed.)

For the profit-maximization problem of an attention seeking firm, given by (3.2) and (3.10), nothing changes since $\tau$ and thus $z_{0}$ and $\widetilde{\varepsilon}$ are exogenous to the single firm. Also 
the functions $\sigma^{*}(r)$ and $\kappa(r)$ determined by Lemma 1 remain unchanged since the condition that marginal and average signal cost coincide under free entry (Equation (A.3)) does not depend on the price elasticity of demand. However, the equilibrium value for signal strength in an information-rich economy changes under (8.2), since a lower price elasticity, allowing to extract more profits from buyers, intensifies the competition for attention. Substituting (8.1) for $z_{0}$ into (8.2) and then $\widetilde{\varepsilon}\left(z_{0}\right)$ for $\varepsilon$ into (A.1), we obtain for equilibrium signal exposure $\widetilde{\tau}$ and minimal signal strength $\widetilde{\sigma}_{\text {min }}$ required in an information-rich economy ${ }^{20}$

$$
\begin{gathered}
\widetilde{\tau}=\tau\left(\frac{\tau}{\tau_{0}}\right)^{\frac{\beta \eta}{1-\beta \eta}}, \\
\widetilde{\sigma}_{\min }=\sigma_{\min }\left(\frac{\tau}{\tau_{0}}\right)^{\frac{\beta \eta}{1-\beta \eta}},
\end{gathered}
$$

where $\tau=\frac{r y}{\kappa(r) \varepsilon}$ is the signal exposure resulting according to Proposition 2 when psychological impact does not feed back on the buyers' willingness to pay.

Note first that $\widetilde{\tau}>\tau_{0}$ if and only if $\tau>\tau_{0}$, since $0 \leq \beta \eta<1$. Thus, the criterion of Proposition 1 for determining whether an economy is information-rich or information-poor is unchanged. Only the economic fundamentals discussed in Section 5.1 matter for the scarcity regime. But as soon as the economy crosses the frontier of information-richness, scarcity of attention is amplified by the interaction of the economic fundamentals (given by $\left.\tau / \tau_{0}\right)$ and the psychological impact on demand behavior captured by $\beta \eta$. The factor of magnification $\left(\tau / \tau_{0}\right)^{\frac{\beta \eta}{1-\beta \eta}}$ increases with the degree of information-richness $\tau / \tau_{0}$ and with the strength of the psychological feedback mechanism $\beta \eta$.

Simultaneously with the magnification of signal exposure and minimal signal strength the realized price-cost margin rises from $\frac{p-c}{p}=\frac{1}{\varepsilon}$ to

$$
\frac{p-c}{p}=\frac{1}{\widetilde{\varepsilon}}=\frac{1}{\varepsilon}\left(\frac{\tau}{\tau_{0}}\right)^{\frac{\beta \eta}{1-\beta \eta}}
$$

\footnotetext{
${ }^{20}(2.5)$ together with (3.4) and (3.5) imply $\widetilde{\tau}=\widetilde{\sigma}_{\min } M$. Thus, according to (A.1), $\widetilde{\tau}=\widetilde{\sigma}_{\min } M=$ $\frac{r y}{\varepsilon\left(z_{0}\right) \kappa(r)}$ which with (8.1), (8.2) reduces to $\widetilde{\tau}=\frac{r y}{\varepsilon \kappa(r)}\left(\frac{\widetilde{\tau}}{\tau_{0}}\right)^{\beta \eta}$. This gives (8.3) and, due to the fact that $M=\tau_{0}$ in an information-rich economy, also (8.4).
} 
(Substitute $\widetilde{\tau}$ for $\tau$ in (8.1) and use (8.2)).

Thus, price-cost margins are higher in markets with strong competition for attention. If attention seeking is intensified in reaction to attractive economic fundamentals $(\tau)$, the items which succeed in entering a buyer's mind, enter with higher strength. If this persuades the buyers that they need the different items more strongly than the original value of $\varepsilon$ suggested, firms can extract more money per item. This makes attention seeking even more attractive and reinforces the competition for attention and its psychological impact.

This magnification effect can have far-reaching macroeconomic consequences. One implication of (8.5) is that the aggregate level of resources allocated to attention-seeking activities rises. According to the equilibrium analysis in Section 4, the cost of attention seeking incurred by each firm is $C\left(\sigma^{*}(r), r\right)$ and $T=\frac{R y}{\varepsilon C\left(\sigma^{*}, r\right)}$ firms enter. Thus, total attention seeking cost are given by $R y / \varepsilon$. If the behavioral magnification effect $(\beta \eta>0)$ is at work, the cost rises to $\frac{R y}{\varepsilon}\left(\frac{\tau}{\tau_{0}}\right)^{\frac{\beta \eta}{1-\beta \eta}}$.

Another implication of (8.5) concerns real wages. Consider an economy in which labor is the only input in the production of goods as well as in the generation and distribution of signals. Suppose that each individual is endowed with one unit of labor. Then, $y=w$ and $c=w / A$ where $w$ is the nominal wage rate and $A$ is labor productivity in production. Moreover, $\tau=\frac{r}{\kappa(r) \varepsilon}$ where $\kappa(r)$ is measured in units of labor, i.e. $\kappa(r)=C_{\sigma^{*}}\left(\sigma^{*}(r), r\right) / w$. Real per-capita income is given by the real wage rate $w / p=A(1-1 / \widetilde{\varepsilon})$ which in view of $(8.5)$ amounts to

$$
\frac{w}{p}=A\left(1-\frac{1}{\varepsilon}\left(\frac{\tau}{\tau_{0}}\right)^{\frac{\beta \eta}{1-\beta \eta}}\right) .
$$

Thus, if consumers are seduced by the psychological impact of intensified attention seeking (so that $\beta \eta>0$ ), changes in the economic fundamentals which increase the degree of information-richness $\left(\tau / \tau_{0}\right)$ lower real wages. Since the incentives for attention seeking are magnified, resources are shifted from production to competition for attention and 
price-cost margins are blown up. This leaves less for the workers' consumption. The conclusion only holds under the assumption that labor productivity $A$ does not rise with the increase of $\tau / \tau_{0}$. As discussed in Section 5, IT-progress and international integration are the main economic causes of rising scarcity of attention, reflected in an increase in $\tau=\frac{r}{\kappa(r) \varepsilon}$. Another cause was media-intermediation considered in Section 7. Whether they are good or harmful for real wages depends on how strongly they improve labor productivity in production in addition to their effect on intensifying the competition for attention.

\subsection{Psychological impact and sectoral economic structure}

In an economy with more than one sector consumers have to decide on the budget they allocate on the goods in the different sectors. This points to another channel for the interaction between psychological impact and economic fundamentals.

Suppose that utility $U$ considered so far enters the representative consumer's utility function $\mathcal{U}(U, V)$ as a subutility index in addition to quantity $V$ purchased from a second sector at price $p_{v}$. To keep things simple, assume

$$
\mathcal{U}(U, V)=U^{\gamma} V^{1-\gamma}, \quad 0<\gamma<1
$$

Then, the budget spent on the $M$ items in the $U$-sector is given by

$$
y_{U}=\gamma y
$$

Suppose that the two sectors differ with respect to the problem of limited attention. For instance, let $V$ be a good of which everybody is aware from birth so that no information processing is involved in the $V$-sector, whereas in the $U$-sector we have competition under limited attention. Using (8.8) in Proposition 2, we get for equilibrium signal exposure

$$
\tau=\frac{r \gamma y}{\kappa(r) \varepsilon}
$$


which implies psychological impact $z_{0}=\left(\tau / \tau_{0}\right)^{\beta}>1$ in an information-rich economy. If $\tau$ rises, the $M\left(=\tau_{0}\right)$ items of the $U$-sector a consumer is aware of are brought to the consumer's attention with more signal strength and are therefore more strongly on her or his mind. This may induce a shift in consumer preferences in favor of the $U$-sector. In (8.7) such a psychological effect can be captured by relating $\gamma$ to $z_{0}$.

$$
\widetilde{\gamma}\left(z_{0}\right)=\gamma z_{0}^{\delta}, \quad 0 \leq \delta<\beta
$$

Using $\widetilde{\gamma}$ instead of $\gamma$ in $(8.9)$, we get $\widetilde{\tau}=\tau\left(\tau / \tau_{0}\right)^{\frac{\delta \beta}{1-\delta \beta}}$ and $\widetilde{z}_{0}=\left(\tau / \tau_{0}\right)^{\frac{\beta}{1-\delta \beta}} \cdot{ }^{21}$ And the budget spent on the $U$-sector increases from $y_{U}=\gamma y$ to

$$
\widetilde{y}_{U}=\left(\tau / \tau_{0}\right)^{\frac{\delta \beta}{1-\delta \beta}} \gamma y
$$

For $\delta \beta>0$, a rise in the incentives for attention seeking in the $U$-sector - caused for instance by IT-progress, access to media or international markets - is magnified and the sectoral structure of the economy gets biased towards the sector prone to intensified attention grabbing.

\subsection{Psychological impact and labor supply}

The two-sector analysis of Section 8.2 easily carries over to labor supply. Let $V=H-h$ be leisure, where $h$ is labor time and $H$ is total time. Then $y_{U}=w h$ and $U=M^{\frac{1}{\varphi}} \frac{y_{U}}{p M}=$ $M^{\frac{1-\varphi}{\varphi}} \frac{w h(\varepsilon-1)}{\varepsilon C}$. Maximizing $\mathcal{U}=U^{\gamma}(H-h)^{1-\gamma}$, we obtain

$$
h=\gamma H, \quad y_{U}=w \gamma H, \quad \tau=\frac{r w \gamma H}{\kappa(r) \varepsilon} .
$$

Assuming again $\widetilde{\gamma}\left(z_{0}\right)=\gamma z_{0}^{\delta}$, we get $\widetilde{z}_{0}=\left(\tau / \tau_{0}\right)^{\frac{\beta}{1-\delta \beta}}$ in the equilibrium of an information-rich economy and thus

$$
\widetilde{h}=\left(\frac{\tau}{\tau_{0}}\right)^{\frac{\delta \beta}{1-\delta \beta}} \gamma H .
$$

\footnotetext{
${ }^{21}$ Note again that $\widetilde{\tau}>\tau_{0}$ if and only if $\tau>\tau_{0}$ so that the criterion for the scarcity regime is not changed by this psychological magnification effect.
} 
An increase in $r / \kappa(r)$ intensifies the competition for attention so that consumer goods are brought more strongly to the consumers' attention. If they react by attributing to consumption higher subjective valuation so that $\beta \delta>0$, then they increase labor supply to earn more money. This increase in consumer budgets induces a further increase of attention seeking activities. In sum, individuals become more consumption- and workoriented if in an already information-rich economy IT-progress, international integration or media channels with global range intensify the competition for attention. As long as an economy is information-poor, no such consequences are to be expected.

\section{Conclusion}

The purpose of this paper was to elaborate the implications of basic empirical facts of attention and perception psychology for competitive equilibrium and important macroeconomic variables. By combining psychological evidence on limited attention with the standard model of monopolistic competition, we have got a psychologically founded framework for the interplay of scarcity of attention and conventional economic scarcity. Competition for the buyers' money is embedded in the competition for their attention. The nature of the competition for attention depends on a psychological parameter $\left(\tau_{0}\right)$ representing the mental capacity of the representative buyer and on the economic fundamentals determining the firms' incentives for attention seeking. The following fundamentals have been identified as the factors which are decisive for whether an economy is information-rich or information-poor: (i) IT-progress allowing firms to address a wider range of buyers or lowering the cost of addressing a given range. (ii) An increase in market size, for instance, by international integration allowing attention seeking across borders. (iii) The possibility to expand attention seeking from direct interaction to media channels with global range.

At the positive level, the analysis leads to concrete predictions on how the economic equilibrium changes, if technical progress, globalization or media-intermediation move the economy from an information-poor state to information-richness with scarcity of attention. In addition to the familiar variables of the monopolistic competition model, the 
following variables are characterized: (i) Equilibrium diversity in an information-poor and information-rich economy, respectively. In particular, the total number of produced items, the number of items per firm, the number of items perceived and consumed by an individual and cultural distinction (measuring the overlap of items in the choice sets of different individuals). (ii) The level of attention seeking activities. In particular, the minimal signal strength required to be attended by buyers, the cost of attention seeking incurred by firms to get on the buyers' mind, and total signal exposure of buyers.

At the normative level, welfare, efficiency and possible policy interventions are characterized. In an information-poor economy individuals gain when IT-progress, international integration or media increase the firms' incentives to bring their products to the attention of buyers, since there is lack of information rather than scarcity of attention. As a consequence, welfare in an information-rich economy is definitely higher than in an informationpoor economy. However, whereas in the information-poor economy decentralized competition for attention and money leads to an efficient equilibrium, in an information-rich economy the decentralized equilibrium is inefficient. The reason is that there is wasteful competition for scarce attention. An efficient solution can be implemented by imposing a linear tax on attention seeking activities and distributing the raised revenue to the buyers. A characterization of the optimal tax rate was provided.

Finally, behavioral effects of the psychological impact of attention seeking were considered. If buyers are seduced by the attention-seeking activities of firms so that their subjective valuation of items rises when the items are brought to their attention more strongly, changes in the factors responsible for information-richness (IT-progress, globalization, media mediation) are amplified. The following implications of this interaction between psychological impact of attention seeking and economic determinants of information-richness have been analyzed: (i) In an information-rich economy price-cost margins rise with the strength of attention seeking activities.(ii) If the factors which lead to stronger competition of attention do not increase labor productivity in production at the same time, real wages deteriorate. (iii) In a multisectoral model, the sectoral economic 
structure is biased in favor of industries prone to strong competition for attention. (iv) Individuals become more consumption- and work-oriented when changes in the economic fundamentals increase information-richness and thus intensify the competition for scarce attention. In an information-poor economy none of these behavioral magnification effects is at work.

\section{Appendix}

Proof of Lemma 1. Substituting (3.10) for $\pi\left(q_{t}\right)$ in (3.2), we have $\Pi\left(q_{t}\right)=r q_{t} \frac{y}{\varepsilon M}-$ $C\left(q \sigma_{\min }, r\right)$. Thus, first-order condition for $\max _{q} \Pi(q)$ and zero-profit condition $\Pi\left(q^{*}\right)=0$ read:

$$
\begin{gathered}
\frac{r y}{\varepsilon M}-\sigma_{\min } C_{\sigma}\left(q^{*} \sigma_{\min }, r\right)=0, \\
\frac{q^{*} r y}{\varepsilon M}-C\left(q^{*} \sigma_{\min }, r\right)=0,
\end{gathered}
$$

respectively. (The second-order condition $\Pi^{\prime \prime}<0$ is guaranteed by $C_{\sigma \sigma}>0$.) Combining (A.1), (A.2) and setting $\sigma^{*}=q^{*} \sigma_{\min }$, we get the equation

$$
C\left(\sigma^{*}, r\right)-\sigma^{*}(r) C_{\sigma}\left(\sigma^{*}, r\right)=0
$$

which defines a function $\sigma^{*}(r)$ with $\frac{d \sigma^{*}}{d r}=\frac{C_{r}-\sigma^{*} C_{\sigma r}}{\sigma^{*} C_{\sigma \sigma}}$.

For specification (4.3), equation (A.3) reduces to $f(r)-\frac{1}{2} f \sigma^{* 2}=0$ which gives $\sigma^{*}(r)=\sqrt{\frac{2 f(r)}{d}}$. QED.

\section{Proof of Proposition 1 and 2.}

a) Characterization of $T$ : Substitute (4.2) for $M$ in (A.2) and solve for $T$ to get $T=$ $\frac{R y}{\varepsilon C\left(\sigma^{*}(r), r\right)}$. For $(4.3), C\left(\sigma^{*}(r), r\right)=2 f(r)$ since $\sigma^{*}(r)=\sqrt{2 f(r) / d}$ according to Lemma 1. b) Characterization of $\tau$ : Substitute (3.3) for $M$ in $\tau=M \sigma_{\min }$ and use then $\sigma_{\min }=$ $\sigma^{*}(r) / q^{*}$ from (4.4) to get $\tau=r T \sigma^{*}(r) / R$. With $T$ from part a) and $C\left(\sigma^{*}(r), r\right) / \sigma^{*}(r)=$ $C_{\sigma}\left(\sigma^{*}(r), r\right) \equiv \kappa(r)$ from (A.3) we get $\tau=\frac{r y}{\varepsilon \kappa(r)}$. Thus, $\tau>\tau_{0}$ is equivalent to (4.5). Corollary 1 follows from the fact that $\kappa(r)=d \sigma^{*}(r)=\sqrt{2 d f(r)}$. QED. 
Proof of Proposition 3. $\sigma_{\min }=1$, according to (3.4). This together with (4.4) implies $q^{*}=\sigma^{*}(r)$. Moreover, according to (3.4), $M=\tau$ where $\tau$ is given by Proposition 2 . Substitute this for $M$ in (3.7) to get $S$. QED.

Proof of Proposition 4. $M=\tau_{0}$ according to (3.5). This together with (4.2) and $T$ from Proposition 2 implies $q^{*}=\frac{\tau_{0 \varepsilon C}\left(\sigma^{*}(r), r\right)}{r y}$. With $T$ and $q^{*}, S$ follows from (4.2). Substitute $q^{*}$ into (4.4) and use (A.3) to get $\sigma_{\min }=\frac{r y}{\tau_{0 \varepsilon \kappa(r)}}$. Then $\tau$ follows from $\tau=M \sigma_{\min }$. For the Corollary use again $C\left(\sigma^{*}(r), r\right)=2 f(r)$ and $\kappa(r)=\sqrt{2 d f(r)}$. QED.

Proof of Proposition 5. If $I$ is IP then $H$ and $F$ are IP, since $\tau_{I}=\tau_{H}+\tau_{F}<\tau_{0}$ implies $\tau_{k}<\tau_{0}$. In this case, according to Proposition 3, $S_{I}=M_{I}=\tau_{I}\left(=\tau_{H}+\tau_{F}\right)$ and $S_{k}=M_{k}=\tau_{k}$. Thus $S_{k}=M_{k}<M_{I}=S_{I}=S_{H}+S_{F}$. If $H$ and $F$ are IP and $I$ is IR, then, according to Proposition 3 and $4, M_{k}=S_{k}=\tau_{k}<\tau_{0}=M_{I}=S_{I}$ and $S_{H}+S_{F}=\tau_{H}+\tau_{F}>\tau_{0}$. Thus, $S_{k}=M_{k}<M_{I}=S_{I}<S_{H}+S_{F}$. If $k$ and $I$ are IR, then $S_{k}=M_{k}=M_{I}=S_{I}=\tau_{0}$ and $S_{I}=\tau_{0}<S_{k}+S_{k^{\prime}}=\tau_{0}+\min \left\{\tau_{0}, \tau_{k^{\prime}}\right\}$. This proves the claims in Part (i) and (ii). Part (iii) follows from (3.7) and the fact that $\rho \geq R_{H}+R_{F}$ implies $r_{k}=\min \left\{\rho, R_{k}\right\}=R_{k}, r_{I}=\min \left\{\rho, R_{H}+R_{F}\right\}=R_{H}+R_{F}$. Thus, $\frac{S_{k}}{M_{k}}=\frac{S_{I}}{M_{I}}=1$ and $\frac{S_{H}+S_{F}}{M_{k}}=1+\frac{S_{k^{\prime}}}{M_{k}}>1$. QED.

Proof of Proposition 6. Substituting (6.8) for $x$ in (6.6) and combining the result with (6.5) and (6.7), we obtain

$$
y^{n}=\frac{p}{c}\left[y-\frac{C\left(q \sigma_{\min }, r\right)}{q r} M\right]
$$

where $M R / r=S$ from (3.7) and $S=q T$ from (4.2) have been used. Thus, the planner's problem is

$$
\max _{\sigma_{\min }, q, M} U=M^{\frac{1}{\varphi}-1}\left[y-\frac{C\left(q \sigma_{\min }, r\right)}{q r} M\right]
$$

subject to the perception constraints (3.4) and (3.5) implied by gating mechanism (2.5). 
$C_{\sigma}>0$ and $\sigma_{\min } \geq 1$ immediately imply $\sigma_{\min }=1$ for optimal signal strength. And the first-order condition for $q$ coincides with (A.3) so that $q \sigma_{\min }=\sigma^{*}(r)$. This proves Part (i). Differentiating (A.5) with respect to $M$, we get the first-order condition $\left(\frac{1}{\varphi}-1\right) M^{\frac{1}{\varphi}-2}$ $\left[y-\frac{C}{q r} M\right]-M^{\frac{1}{\varphi}-1} \frac{C}{q r}=0$ which reduces to

$$
M^{\frac{1}{\varphi}-2}\left[\left(\frac{1}{\varphi}-1\right) y-\frac{1}{\varphi} \frac{C}{q r} M\right]=0 .
$$

This gives us

$$
M^{*}=\min \left\{(1-\varphi) y \frac{q r}{C\left(\sigma^{*}(r), r\right)}, \tau_{0}\right\},
$$

since $M \leq \tau_{0}$ according to (3.4), (3.5). The second-order condition reads $\left(\frac{1}{\varphi}-1\right)\left(\frac{1}{\varphi}-2\right)$ $M^{\frac{1}{\varphi}-3}\left(y-\frac{C}{q r} M\right)-2\left(\frac{1}{\varphi}-1\right) M^{\frac{1}{\varphi}-2} \frac{C}{q r}<0$ which is equivalent to $(1-2 \varphi)\left(y-M \frac{C}{q r}\right)<$ $2 \varphi M \frac{C}{q r}$. This inequality reduces to $(1-2 \varphi) y \frac{q r}{C}<M$ which certainly holds at $M$ determined by (A.6). This, $q=\sigma^{*}(r)$ for $\sigma_{\min }=1$ and the fact that $\kappa(r)=C_{\sigma}\left(\sigma^{*}(r), r\right)=$ $C\left(\sigma^{*}(r), r\right) / \sigma^{*}(r)$ prove Part (ii) and Part (iii). Part (iv) follows immediately from (A.4), (A.5). QED.

Proof of Proposition 7. Combining (6.8) with (6.6) and using $S=M R / r$, we get for the gross-profit of a firm bringing $q_{t}$ items to the attention of $r$ buyers: $r q_{t} \vartheta y^{n} / M$, where $\vartheta \equiv(p-c) / p$. Thus, net profits for a firm facing $\sigma_{\min }$ and $\operatorname{tax} B=B_{0} \sigma_{t}, \sigma_{t}=q_{t} \sigma_{\min }$, are given by $\Pi\left(q_{t}\right)=r q_{t} \vartheta y^{n} / M-C\left(q_{t} \sigma_{\min }, r\right)-B_{0} q_{t} \sigma_{\min }$. Instead of (A.1), (A.2) we get

$$
\begin{aligned}
& \frac{r \vartheta y^{n}}{M}-\sigma_{\min }\left[C_{\sigma}\left(q \sigma_{\min }, r\right)+B_{0}\right]=0 \\
& \frac{q r \vartheta y^{n}}{M}-\left[C\left(q \sigma_{\min }, r\right)+B_{0} q \sigma_{\min }\right]=0
\end{aligned}
$$

for first-order and zero-profit condition, respectively. Eliminating $r \vartheta y^{n} / M$, we obtain $C\left(q \sigma_{\min }, r\right)-\sigma_{\min } C_{\sigma}\left(q \sigma_{\min }, r\right)=0$, which is identical to equation (A.3). Thus, $\sigma_{t}=$ $\sigma^{*}(r)$ as defined by Lemma 1 (and efficient according to Proposition 6). Distributing the resulting tax revenue $B_{0} \sigma^{*}(r) T$ to the $R$ buyers, we have the net income $y^{n}=$ $y+B_{0} \sigma^{*}(r) T / R$ which with $T=S / q=\frac{M R}{r q}($ see $(4.2))$ and $\sigma^{*}(r)=q \sigma_{\min }$ reduces to

$$
y^{n}=y+B_{0} \sigma_{\min } M / r .
$$


Since $\tau=M \sigma_{\min }$, according to (2.5), (3.4) and (3.5), condition (A.8) implies $\tau=$

$\frac{r \vartheta y^{n}}{\kappa(r)+B_{0}}$ which after substitution of (A.10) reduces to $\tau=\frac{\vartheta\left(r y+B_{0} \tau\right)}{\kappa(r)+B_{0}}$. Solving this equation for $\tau$, we get $\tau=\frac{\vartheta r y}{\kappa+(1-\vartheta) B_{0}}$ which for $B_{0}$ given by (6.11) reduces to $\tau=\tau_{0}$. Hence, $\sigma_{\min } M=\tau_{0}$ in equilibrium. According to (2.5) and (3.5), this is only consistent with the perceptual filter if $\sigma_{\min }=1$ and $M=\tau_{0}$, which is efficient. Finally, with $\sigma_{\min } M=\tau_{0}$, equation (A.10) reduces to $y^{n}=\frac{y-\kappa \tau_{0} / r}{1-\vartheta}$ which gives the efficient level of real disposable income of Proposition 6 , since $1-\vartheta=c / p$ by definition QED.

\section{References}

Camerer, Colin F. [2003], "The behavioral challenge to economics: Understanding normal people", Paper presented at Federal Reserve of Boston meeting.

Caplan, Bryan and Cowen, Tyler [2004], "Do We Underestimate the Benefits of Cultural Competition?", American Economic Review 94, 402-407.

Davenport, Thomas H., and Beck, John C. [2001], The Attention Economy, Boston/Mass: Harvard Business School Press.

Dixit, Avanish K., and Stiglitz, Joseph E. [1977], "Monopolistic Competition and Optimum Product Diversity", American Economic Review 67, 297-308.

Dukas, Reuven [2004], "Causes and Consequences of Limited Attention", Brain Behavior and Evolution 63, 197-210.

Falkinger, Josef [2003], "Attention Economies", CESifo Working Paper No. 1079.

Gabaix, Xavier, and Laibson, David [2002], "The 6D Bias and the Equity-Premium Puzzle", in: B. Beranke and K. Rogoff (Eds.), NBER Macroeconomic Annual, 257312.

Gabaix, Xavier, and Laibson, David [2004], "Bounded Rationality and Directed Cognition", MIT and Harvard mimeo. 
Gabaix, Xavier, Laibson, David, Moloche, Guillermo, and Weinberg, Stephen [2003], "The Allocation of Attention", Working Paper 03-31, MIT, Department of Economics, Cambridge/M.

Hirshleifer, David and Teoh, Siew H. [2003], "Limited attention, information disclosure, and financial reporting", Journal of Accounting and Economics 36, December, 337386.

Hirshleifer, David, Lim, Sonya S. and Teoh, Siew H. [2002], "Disclosure to a Credulous Audience: The Role of Limited Attention", Ohio State University.

Kahneman, Daniel [1973], Attention and Effort, New Jersey: Prentice Hall.

Klamer, Arjo and Van Dalen, Hendrik P. [2002] "Attention and the art of scientific publishing", Journal of Economic Methodology 9, 289-315.

Kraut, Robert E., Sunder, Shyam, Morris, James, Telang, Rahul, Filer, Darrin and Cronin, Matt. [2002], "Markets for Attention: Will Postage for Email Help?", http://basic.fluid.cs.cmu.edu/articles/kraut02.

Lachter, Joel, Forster, Kenneth I., and Ruthruff, Eric [in Press], "Forty-five Years After Broadbent: Still No Identification Without Attention", Psychological Review.

Luce, R. Duncan [2004], "A Way to Blend Fechner and Stevens", Irvine mimeo.

Pashler, Harold E. [1998], The Psychology of Attention, Cambridge/M.: MIT Press.

Reis, Ricardo [2004 a], "Inattentive Consumers", NBER Working Paper 10883.

Reis, Ricardo [2004 b], "Inattentive Producers", unpublished manuscript, Harvard University.

Shapiro, Carl, and Varian, Hal R. [1999], Information Rules. A Strategic Guide to the Network Economy. Boston/M.: Harvard Business School Press. 
Simon, Herbert A. [1971], "Designing Organizations for an Information-rich World", in: Greenberger, M. (Ed.), Computers, Communications, and the Public Interest, pp. 38-52, Baltimore: John Hopkins Press. (Cited after reprint in: H.A. Simon [1982], Models of bounded rationality. Volume 2: Behavioral Economics and Business Organization, Cambridge/M.: MIT Press.)

Sims, Christopher A. [2003], "Implications of rational inattention", Journal of Monetary Economics 50, 665-690.

Stevens, S.S. [1986], Psychophysics. Introduction to Its Percepteual, Neural, and Social Prospects, New Brunswick and Oxford: Transcation Books. (Originally published: New York: Wiley 1975.) 
Information-poor economy:

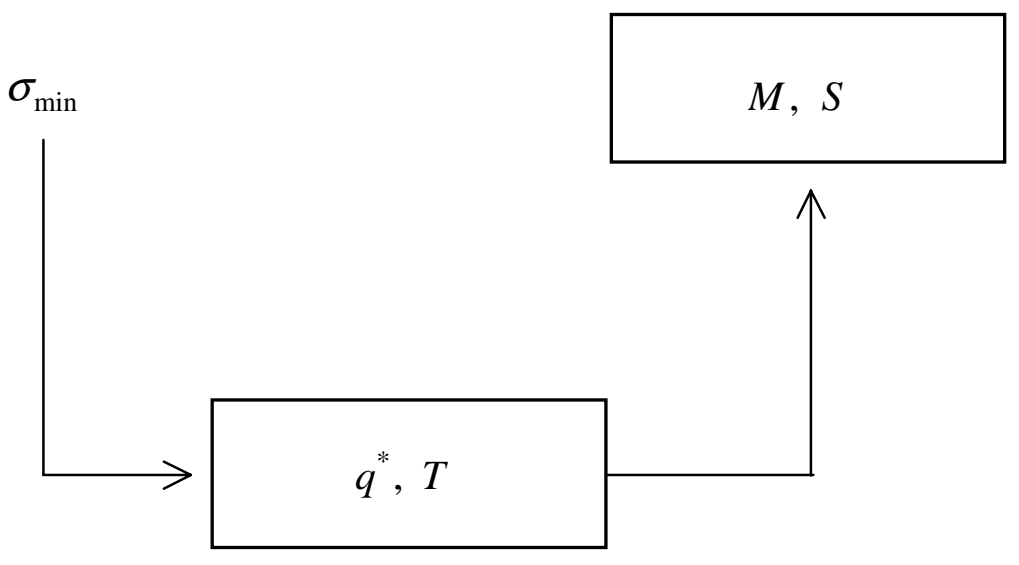

\section{Information-rich economy:}

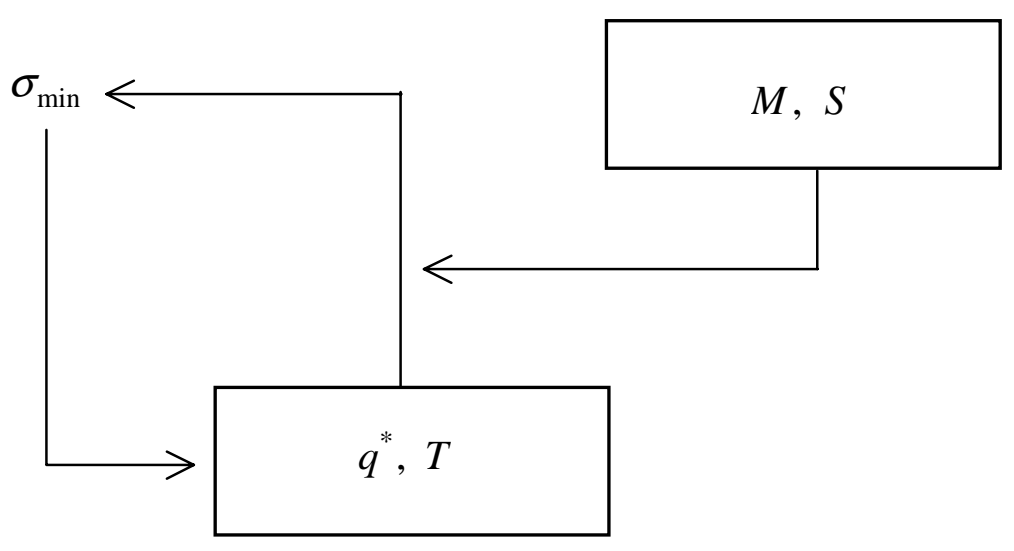

Figure 1: Embedding of economic competition in perception filter. 


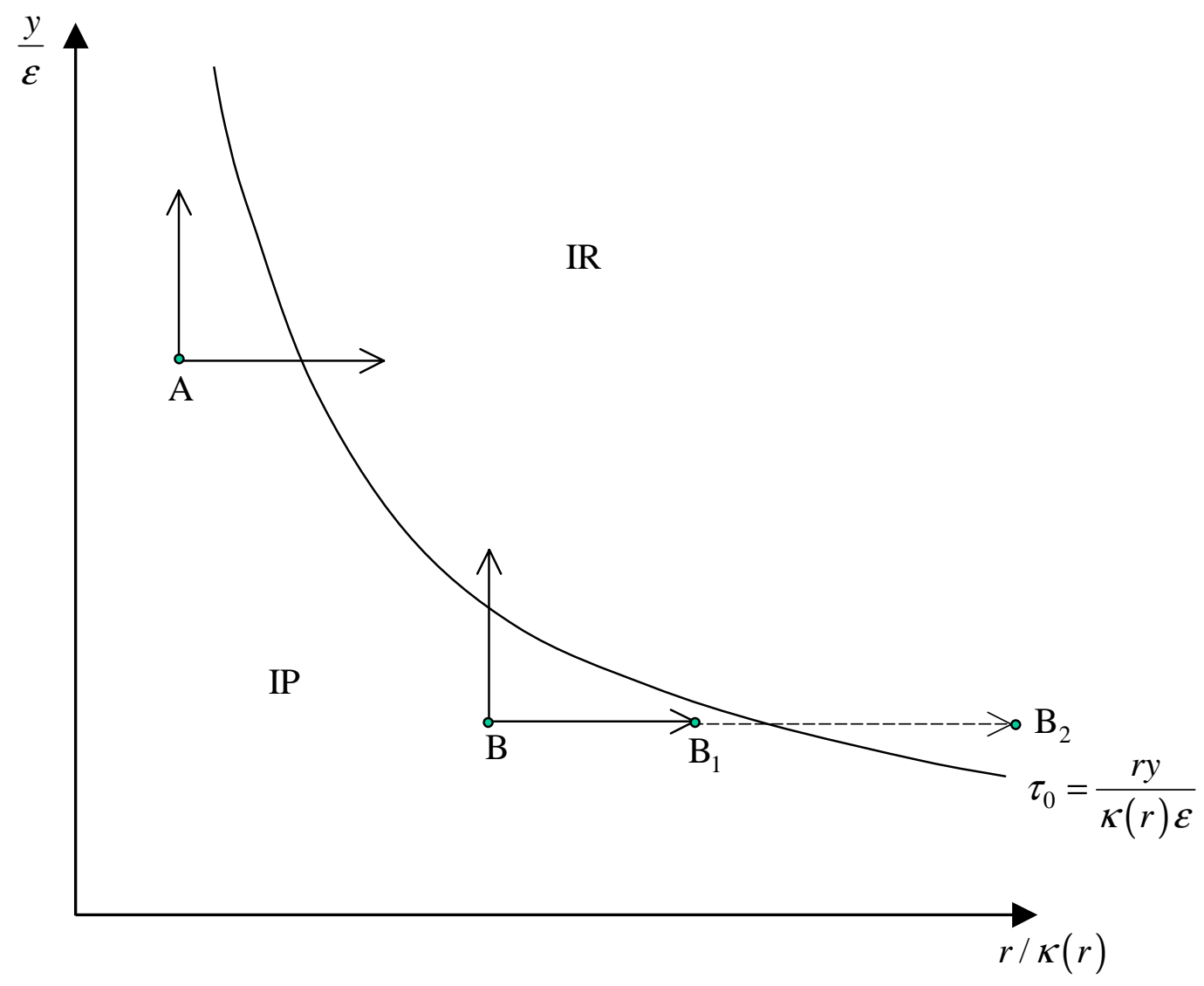

Figure 2: Frontier to information-rich economy 


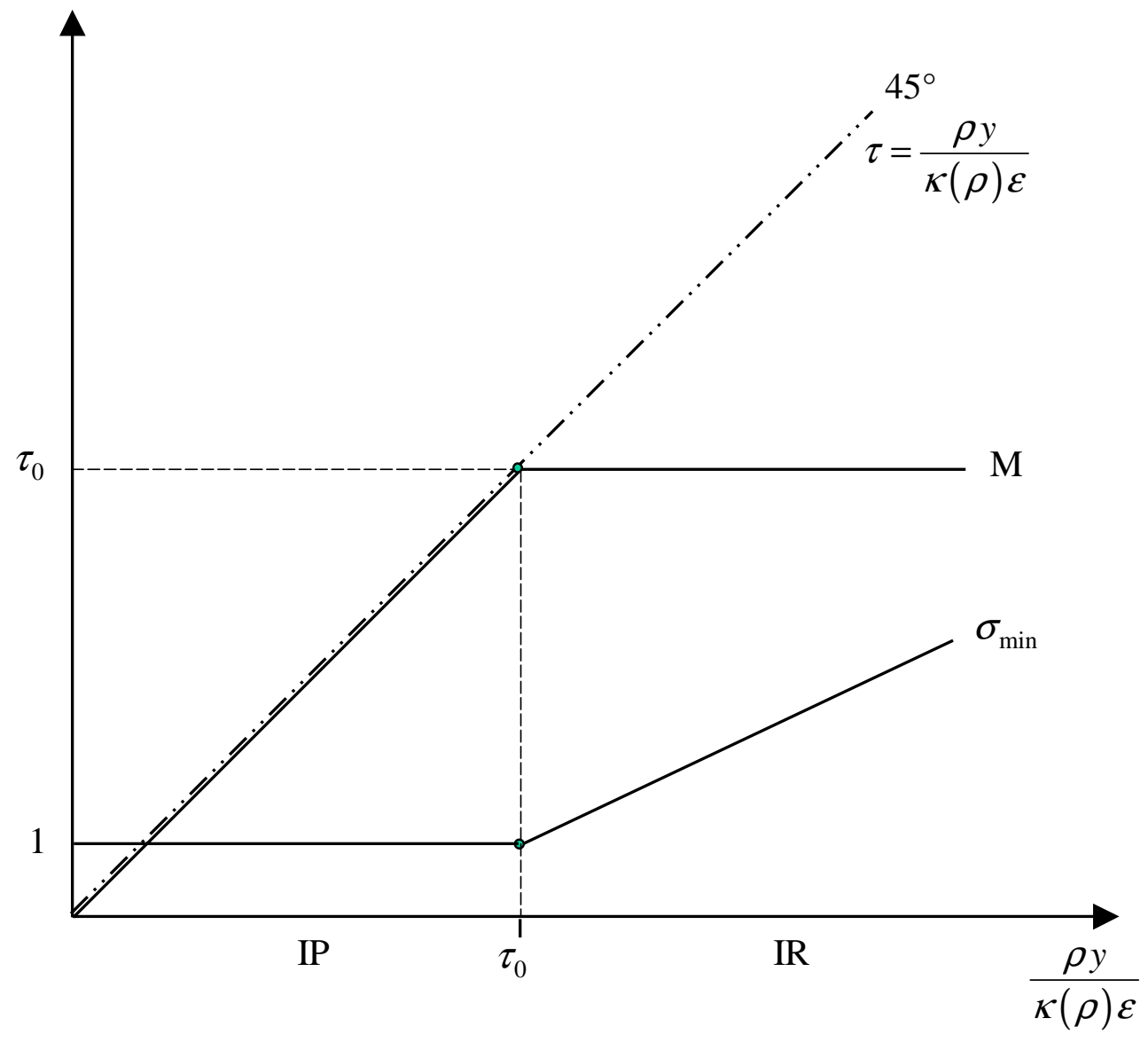

Figure 3: Impact of economic fundamentals on signal strength and local diversity. 